\title{
1 Environmental Metabolomics: Analytical Strategies
}

2 Authors: Melissa C. Kido Soule ${ }^{\mathrm{a}}$, Krista Longnecker ${ }^{\mathrm{a}} \mathbb{I}$, Winifred M. Johnson ${ }^{\mathrm{b}} ¥$,

3 Elizabeth B. Kujawinskia

4 a Woods Hole Oceanographic Institution, Marine Chemistry and Geochemistry, Woods

5 Hole, MA 02543, U.S.A.

$6{ }^{\mathrm{b}}$ MIT/WHOI Joint Program in Oceanography/Applied Ocean Sciences and Engineering,

7 Woods Hole Oceanographic Institution, Marine Chemistry and Geochemistry, Woods

8 Hole, MA 02543, U.S.A.

$9 \quad \ddagger$ msoule@whoi.edu; Ilklongnecker@whoi.edu; ¥wjohnson@whoi.edu;

10 tekujawinski@whoi.edu

11 Running title: Analytical strategies for marine metabolomics

12 For submission to: Marine Chemistry

$13 \ddagger$ Corresponding author. Mailing address: WHOI MS\#4, Woods Hole, MA 02543.

14 Phone: (508) 289-2879. Fax: (508) 457-2164. E-mail: msoule@whoi.edu

15 Keywords: targeted and untargeted metabolomics, LC-MS, dissolved organic matter

\section{ABSTRACT}

Microbial metabolism plays a primary role in shaping the marine carbon cycle through processes of carbon fixation and remineralization. Many metabolic intermediates pass through the reservoir of marine dissolved organic matter (DOM), as compounds move among microbes as part of complex ecological networks of interactions. Environmental metabolomics can be used to identify and quantify these compounds, and thus will provide insight into the chemical underpinnings of microbial networks at the foundation of global biogeochemical cycles. Here we present methods for metabolite profiling (untargeted metabolomics) and for relative quantification (targeted metabolomics) of intracellular and extracellular metabolites from marine microbes. We describe our approach to method development with regard to metabolite extraction and instrumental analysis, culminating in the methods currently in use in our laboratory. 


\section{INTRODUCTION}

Marine microbes play a key role in global biogeochemical cycles through their metabolic interactions with dissolved organic matter (DOM) in the ocean. The suite of

32 compounds produced by a microbe reflects its metabolic state, which is a function of its genetic potential and its environment. Interactions with neighboring organisms, the availability of nutrients, and environmental conditions all exert an influence on the expressed metabolic profile and hence on microbial contributions to the DOM pool (Carlson, 2002; Nagata, 2000). Heterotrophic and many autotrophic microorganisms

37 also rely on the low molecular weight $(<600 \mathrm{Da})$ fraction of DOM for their growth and metabolism (reviewed in Kujawinski, 2011). Identifying metabolites that pass through the pool of DOM and that interconnect microbial species is an important step in understanding the impact of microbes on the global carbon cycle. New insights into

41 microbe-DOM interactions within the marine ecosystem can be gained through the use 42 of tools from the field of metabolomics.

44 biological system (e.g. cells, tissues, or biofluid) (Viant, 2007). The purpose of environmental metabolomics is the characterization of an organism's metabolic response to natural or anthropogenic stressors in its environment (Viant, 2007).

47 Metabolomics experiments generally fall into two categories, untargeted and targeted. 
48 Untargeted metabolomics seeks to detect and describe as much of the metabolome as

49 possible without bias (Patti et al., 2012). By contrast, targeted metabolomics studies are

50 typically focused on quantifying a specified set of compounds through the use of

51 authentic standards ( $\mathrm{Lu}$ et al., 2008). The suite of target compounds is often derived

52 from one or more metabolic pathways of the biological system of interest (Patti et al.,

53 2012).

54 Metabolomics methods can be applied to the study of microbial communities in

55 their environment, including in marine systems. Here, untargeted metabolomics

56 enables the discovery of new or unexpected metabolites, thereby expanding the

57 metabolic pathways that can be queried in microbial consortia. For example, analysis of

58 metabolites exuded by microorganisms into the surrounding dissolved phase (i.e.

59 extracellular metabolites) can be used to elucidate cooperative relationships among

60 microbial consortia members. Targeted metabolomics provides an important

61 quantitative complement to untargeted methods; and in environmental applications,

62 many of these targets are not currently studied in standard organic geochemistry

63 investigations. For example, quantitative profiles of intracellular metabolites from

64 targeted metabolomics can provide a snapshot of how microorganisms respond to

65 environmental stress, such as nutrient limitation. 
The application of metabolomics methods to the marine environment is, however,

67 still in its infancy. In contrast to the large number of metabolomics studies in plants

68 (Quanbeck et al., 2012; Tolstikov and Fiehn, 2002), human systems (Dunn et al., 2008;

69 Kind et al., 2007; Want et al., 2010; Wikoff et al., 2009), and model microorganisms (e.g.

70 yeast or Escherichia coli, Bajad et al., 2006; Lu et al., 2010; Rabinowitz and Kimball, 2007;

71 Yanes et al., 2011), fewer metabolomics profiles have been collected for marine

72 microbes. Studies to date include measurements of specific metabolite classes in

73 seawater, such as B vitamins (Heal et al., 2014; Sañudo-Wilhelmy et al., 2012), as well as

74 broader assessments of primary and secondary metabolites within marine microbial

75 cultures (Baran et al., 2010; Bennette et al., 2011; Fiore et al., in press; Longnecker et al.,

76 2015; Paul et al., 2009; Romano et al., 2014).

77 Marine environmental metabolomics follows in the footsteps of seminal organic

78 geochemistry studies that explored the sources and sinks of biochemicals in the oceans

79 such as amino acids and carbohydrates (Benner, 2002; Lee et al., 2000). These

80

compounds are common to most microbes and participate in numerous metabolic

81 reactions. For example, amino sugars are components of cell walls, peptides and iron-

82 binding ligands (Kaiser and Benner, 2008) and are catabolized by a variety of bacteria in

83 the ocean. The ratios of individual molecules and their stereoisomers track the rate of

84 organic matter degradation during transport to the deep ocean (Kaiser and Benner,

85 2009). Compound-class centric studies, such as these, have provided fundamental 
86 insights to specific research questions within biogeochemistry. With the advent of new

87 analytical approaches, environmental metabolomics can significantly broaden the range

88 of metabolites under study by probing many compound classes simultaneously.

89 Importantly, these complex datasets complement other systems biology tools such as

90 meta-genomics, meta-transcriptomics and meta-proteomics, and together these tools

91 enable systems-wide understanding of microbial metabolism and organic carbon

92 cycling in the environment.

93 A variety of analytical platforms have been used for metabolomics experiments,

94 including liquid (LC) or gas (GC) chromatography coupled with mass spectrometry

95 (MS) (Fiehn, 2008; Lu et al., 2008; Want et al., 2007; Zelena et al., 2009), NMR

96 spectroscopy (Barton et al., 2008), direct infusion MS (Southam et al., 2007), and

97 capillary electrophoresis-MS (Monton and Soga, 2007). Of these, chromatography

98 coupled with MS has emerged as a preferred approach for global metabolic profiling

99 and metabolite quantification (Bennette et al., 2011; Dunn et al., 2011; Patti et al., 2012;

100 Want et al., 2010).

101 Creating a mass-spectrometry based metabolomics method presents several

102 challenges. Metabolites vary widely in their chemical properties and dynamic range,

103 making the selection of a comprehensive extraction protocol and chromatographic

104 separation technique a considerable task. The choice of ionization method can be an 
105 additional source of bias. Ionization techniques such as electrospray ionization (ESI),

106 atmospheric pressure chemical ionization (APCI), and matrix-assisted laser

107 desorption/ionization (MALDI) select for certain analyte physical and chemical

108 properties (Nordström et al., 2008). ESI is particularly well suited for polar metabolites

109 that would normally carry a charge in solution; many metabolites of interest, in fact,

110 have ionizable basic or acidic groups. Many neutral compounds, however, will not

111 ionize efficiently (or at all) with ESI. For example, myo-inositol, a small carbohydrate,

112 does not ionize by ESI. Marine microbial samples pose additional analytical challenges

113 due to the high levels of salt in growth media and in the ocean itself. Salt in the sample

114 matrix can lead to ion suppression of mass spectrometry signal during electrospray

115 ionization, and the build-up of salt over time in the mass spectrometer and ESI source

116 can decrease instrument performance and data quality. Metabolomics methods must

117 also be able to make reproducible measurements, and include strategies to monitor that

118 reproducibility. To this end, the particular strengths of the mass analyzer must be well

119 matched to the experimental goals (i.e. quantitative analysis or global profiling).

120 The purpose of this manuscript is to review our approach over the past five years to

121 the development of methods for marine environmental metabolomics. We present both

122 untargeted and targeted metabolomics methods, in which we extract intracellular and

123 extracellular metabolites from marine microbial samples and measure their composition

124 and relative abundance with ESI coupled to liquid chromatography - mass 
125 spectrometry (LC-ESI-MS). Here, we detail our decisions in developing our analytical strategies with regard to experimental design, metabolite extraction, and LC-MS

127 analysis, culminating with the methods currently in use in our laboratory. We address

128 data analysis methods in a companion paper in this issue (Longnecker et al., in press).

129 2. EXPERIMENTAL SECTION

\subsection{Materials and Chemicals}

Metabolite standards at the highest grade available were obtained through Sigma

132 Aldrich (MO, USA), with the exception of: dimethylsulfoniopropionate (DMSP),

133 purchased from Research Plus Inc. (NJ, USA); 2,3-dihydroxypropane-1-sulfonate

134 (DHPS) and n-acetyltaurine, which were generously donated by Dr. Mary Ann Moran

135 at the University of Georgia; and S-(1,2-dicarboxyethyl)glutathione, purchased from

136 Bachem (CA, USA). Isotopically labeled standards were purchased from Cambridge

137 Isotopes (d3-glutamic acid), from CDN Isotopes (d4-4-hydroxybenzoic acid), and from

138 Toronto Research Chemicals (d5-sodium taurocholate) through Fisher Scientific.

139 Solvents and additives used for metabolite extraction and LC-MS analysis were

140 acetonitrile (Optima LC/MS, Fisher Chemical), methanol (Optima, Fisher Chemical),

141 formic acid (eluent additive for LC/MS, Fluka), hydrochloric acid (Optima, Fisher

142 Chemical), and ammonium hydroxide (ACS Plus, Fisher Chemical). Water purified by a

143 Milli-Q system (Millipore) was used throughout (resistivity $18.2 \mathrm{M} \Omega \bullet \mathrm{cm} @ 25^{\circ} \mathrm{C}$, TOC

$144<1 \mu \mathrm{M})$. 


\subsection{Samples Used for Method Testing} culture media without cells. Filters were stored in cryovials (Nalgene Cryoware ${ }^{\mathrm{TM}}$ ) at $16280^{\circ} \mathrm{C}$ until extraction. The filtrate was acidified to $\mathrm{pH}$ 2-3 with $12 \mathrm{M} \mathrm{HCl}$ and stored at $163 \quad 4^{\circ} \mathrm{C}$ until extraction.

We used a series of laboratory and field samples for testing the metabolomics methods. Marine microorganisms Ruegeria pomeroyi (heterotrophic $\alpha$-proteobacterium), Micromonas pusilla (autotrophic pico-eukaryote), and Thalassiosira pseudonana (autotrophic diatom) were grown in laboratory cultures. Surface seawater samples were collected from the University of Rhode Island's Narragansett Bay Plankton Time Series site. Net trap samples were collected in the Atlantic Ocean during a cruise on the R/V Knorr. Details pertaining to these samples can be found in Table S1 of the Supplemental Information.

\subsection{Metabolite Extractions}

All glassware was acid-washed and combusted at $460^{\circ} \mathrm{C}$ for at least 5 hours. Water samples obtained from laboratory cultures were filtered through $0.2 \mu \mathrm{m}$ PTFE filters (Omnipore, Milipore). Field samples were filtered in series onto $1.6 \mu \mathrm{m}$ glass fiber filters (pre-combusted size GF/A, Fisher Scientific) and $0.2 \mu \mathrm{m}$ PTFE filters. The volume of water filtered typically ranged from 50-4000 mL, depending on anticipated cell abundance (see section 3.2). Filter blanks were used as negative controls by filtering 
Extracellular metabolites were extracted from the acidified filtrate using solid phase

165 extraction (SPE) with 1 g/ 6 cc PPL cartridges (Bond Elut PPL, Agilent), as previously

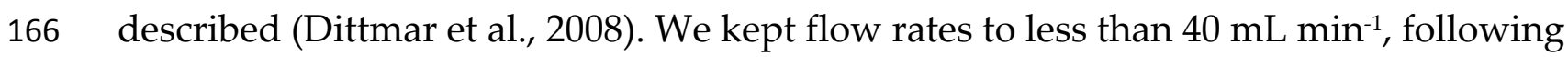

167 the original protocol. We have not tested the effect of flow rate on our extraction

168 efficiencies. The cartridges were rinsed with at least two cartridge volumes of $0.01 \mathrm{M}$

$169 \mathrm{HCl}$ (aqueous solution) and eluted with $8 \mathrm{~mL}$ of methanol. The eluent was reduced to

170 near dryness with vacuum centrifugation. The samples were re-dissolved in $495 \mu \mathrm{L}$ of

$17195: 5$ water:acetonitrile with $5 \mu \mathrm{L}$ of $5 \mu \mathrm{g} / \mathrm{mL}$ of biotin- $\left(\right.$ ring-6,6- $\left.\mathrm{d}_{2}\right)$ and divided into two

172 aliquots to be analyzed with the targeted and untargeted metabolomics methods. The

173 deuterated biotin served as an autosampler injection standard.

$174 \quad$ Intracellular metabolites were extracted from the filters using a method adapted

175 from Rabinowitz and Kimball (2007). Working quickly throughout to minimize

176 degradation, immediately prior to extraction the filters were removed from the freezer,

177 weighed and cut. A combusted aluminum foil surface was used for all weighing and

178 cutting, and scissors and tweezers were rinsed with methanol before use and between

179 samples. Either a portion or the full filter was cut into small pieces and placed at the

180 bottom of an $8 \mathrm{~mL}$ amber glass vial. We determined the amount of filter to extract based

181 on the number of cells filtered (see section 3.2). Cold $\left(-20^{\circ} \mathrm{C}\right)$ extraction solvent was

182 added to each amber vial to adequately cover the filter pieces (500 $\mu \mathrm{L}$ to $1 \mathrm{~mL}$ of

183 40:40:20 acetonitrile: methanol: 0.1M formic acid). In addition, $25 \mu \mathrm{L}$ of a $1 \mu \mathrm{g} / \mathrm{mL}$ 
184 deuterated internal standard mix (d3-glutamic acid, d4-4-hydroxybenzoic acid, and d5185 sodium taurocholate) was added to monitor the intracellular extraction recovery. The 186 vials were gently vortexed to ensure solvent coverage of all filter surfaces and then 187 sonicated for 10 minutes to lyse the cells. Extracts were transferred by pipette to 188 centrifuge tubes (Fisherbrand ${ }^{\mathrm{TM}}$ Premium Microcentrifuge tubes), and the remaining 189 filter pieces were rinsed with $200 \mu \mathrm{L}$ of extraction solvent; the rinse was added to the 190 tubes. The extracts were spun at 20,000xg for 5 minutes at $4^{\circ} \mathrm{C}$ and the supernatant 191 transferred to new $8 \mathrm{~mL}$ amber glass vials without disrupting any particulates at the 192 bottom of the tube (filter or cellular materials). The extracts were neutralized with $6 \mathrm{M}$ 193 ammonium hydroxide and reduced to near dryness using vacuum centrifugation. The 194 intracellular extracts were re-dissolved as described above for the extracellular samples 195 and divided into two aliquots for targeted and untargeted analysis.

196 Additional samples for quality control (QC) were analyzed in all targeted and 197 untargeted experiments. These QC samples included a mix of the authentic metabolite 198 standards described above and a pooled sample consisting of aliquots of each sample.

199 Samples were analyzed in randomized order.

\subsection{Untargeted Metabolomics Instrumentation}

Intracellular and extracellular metabolite samples for untargeted analyses were run

202 within 24-48 hours of extraction using a liquid chromatograph (Thermo Micro AS 
204 Fourier transform ion cyclotron resonance (FT-ICR) mass spectrometer (7T LTQ FT

205 Ultra, Thermo Scientific). Metabolite separation was accomplished on a Synergi Fusion

$206 \mathrm{RP}$ column $(4 \mu \mathrm{m}, 2.0 \times 150 \mathrm{~mm}$, Phenomenex), equipped with a guard column and pre-

207 column filter and maintained at $35^{\circ} \mathrm{C}$ with a Hot Pocket column heater (Thermo

208 Scientific). The column was eluted with (A) $0.1 \%$ formic acid in water and (B) $0.1 \%$

209 formic acid in acetonitrile at a flow rate of $0.25 \mathrm{~mL} \mathrm{~min}^{-1}$. The column was held at 5\% B

210 for $2 \mathrm{~min}$, ramped to $65 \%$ B over $18 \mathrm{~min}$, quickly ramped to $100 \% \mathrm{~B}$ in $5 \mathrm{~min}$, held at

$211 \quad 100 \% \mathrm{~B}$ for $7 \mathrm{~min}$ and then equilibrated at $5 \% \mathrm{~B}$ for $8 \mathrm{~min}$ prior to the next injection

212 (total run time $=40 \mathrm{~min}$ ). Separate autosampler injections of $20 \mu \mathrm{L}$ each were made for

213 positive and negative ion modes.

214 The mass spectrometer was externally calibrated weekly in positive and negative

215 modes using solutions provided by the manufacturer. The capillary temperature and

216 ESI voltage were optimized at $330^{\circ} \mathrm{C}$ and $4.2 \mathrm{kV}$ in positive mode and at $365^{\circ} \mathrm{C}$ and 3.8

$217 \mathrm{kV}$ in negative mode. Sheath gas, auxiliary gas, and sweep gas flow rates were

218 maintained at 35, 5, and 2, respectively (arbitrary units) for both polarities. Both high

219 resolution MS and data dependent MS/MS scans were collected. The MS scan was

220 performed in the FT-ICR analyzer from 100-1000 $\mathrm{m} / \mathrm{z}$, with mass resolving power set to

221 100,000 (defined at $m / z$ 400). In parallel to the FT acquisition, the four most abundant

222 ions in each cycle were fragmented via collision-induced dissociation in the linear ion

223 trap. MS/MS spectra were collected under dynamic exclusion with an exclusion time of 
22420 seconds. Dynamic exclusion allows for greater depth of MS/MS coverage because the

225 instrument will not repeatedly trigger an MS/MS scan of the same ion. Data were

226 collected in profile mode as XCalibur RAW files (XCalibur 2.0.7) and then converted to

227 open source formats using ProteoWizard (Chambers et al., 2012). Each data set may

228 contain thousands of features, where a feature is defined as a unique combination of a

229 mass-to-charge $(\mathrm{m} / \mathrm{z})$ ratio and a retention time. For more details on data processing see

230 Longnecker et al. (in press) in this issue.

$231 \quad 2.5$ Targeted Metabolomics Instrumentation

232 Concentrations of intracellular and extracellular metabolites were measured using

233 an LC system (Thermo Accela Open Autosampler and Accela 1250 Pump) coupled via a

234 heated ESI (H-ESI) source to a triple quadrupole mass spectrometer (Thermo TSQ

235 Vantage). The instrument was calibrated in positive and negative ion modes using a

236 polytyrosine-1,3,6 solution infused directly into the mass spectrometer. Selected

237 reaction monitoring (SRM) conditions for each compound (s-lens, collision energy,

238 parent and fragment ions) were optimized by infusion of authentic metabolite

239 standards. For each metabolite parent mass, quantification and confirmation SRM

240 transitions (a parent and product ion pair) were selected primarily to maximize signal-

241 to-noise. Polarity switching in the H-ESI probe was employed, thereby combining

242 positive and negative SRM transitions in a single run. The SRM transitions were

243 scheduled such that each compound was monitored over a 2-4 min window around its 
244 retention time. This ensured adequate sampling across chromatographic peaks (at least

24510 data points over a 10-24 second baseline peak width). H-ESI parameters were:

246 capillary temperature $275^{\circ} \mathrm{C}$, vaporizer temp $375^{\circ} \mathrm{C}$, sheath gas pressure 40 units, and

247 auxiliary gas pressure 25 units. Spray voltage was $4 \mathrm{kV}$ and $3.2 \mathrm{kV}$ for positive and

248 negative ion modes, respectively. Data was collected in centroid mode as XCalibur

249 RAW files.

250 The LC column, mobile phase and gradient conditions were the same as those used

251 in the untargeted metabolomics analysis. Stock solutions of each metabolite standard

252 were prepared in either Milli-Q water or a 1:1 mix of methanol and water at 1000

$253 \mu \mathrm{g} / \mathrm{mL}$, unless constrained by solubility. Certain standards required the addition of

254 either ammonium hydroxide or formic acid for dissolution in the stock solution. Those

255 that required formic acid include: glutamic acid, guanine, cysteine, riboflavin,

256 guanosine, and aspartic acid. Standards that required ammonium hydroxide include:

257 orotic acid, biotin, xanothosine, folic acid, xanthine, and tyrosine. Stock solutions were

258 stored in the dark at $-20^{\circ} \mathrm{C}$. Prior to metabolomics analysis, individual stock solutions

259 were combined and diluted in Milli-Q water to create 6 metabolite standard mixes each

260 at $10 \mu \mathrm{g} / \mathrm{ml}$; metabolites were grouped together in these mixes based on chemical

261 compatibility. For the targeted method, these 6 mixes were then combined to generate a

262 full mix of all metabolite standards ("standard mix") and diluted in Milli-Q water to

263 concentrations between 0.5 - $1000 \mathrm{ng} / \mathrm{mL}(0.5,1,5,10,25,50,100,250,500$, and 1000 
$264 \mathrm{ng} / \mathrm{mL})$. The current method does not use isotopically-labeled internal standards for 265 each target analyte to account for matrix effects, but rather uses external calibration for

266 relative quantification. External calibration curves for each compound were generated

267 by plotting peak area against concentration for each metabolite standard, and then used 268 to determine relative metabolite concentrations in the intracellular and extracellular 269 extracts.

271 3. RESULTS AND DISCUSSION

272 In this section we discuss key issues involved in several aspects of our marine 273 metabolomics methods: experimental design, sample collection, metabolite extraction, 274 and LC-MS analysis.

\section{3.1 Experimental design:}

276 In designing a metabolomics experiment it is essential to incorporate strategies for 277 assessing data quality and measuring analytical system variability. For this purpose, we 278 include in every study (targeted or untargeted) several types of quality control (QC) 279 samples: pooled samples, standard test mixes, cell-free controls (i.e. growth media 280 without cells processed identically to culture samples), and biological replicates. We 281 also include internal standards for monitoring metabolite extraction and autosampler 282 injection. 

and serves two main purposes. First, it is used to condition the analytical system, in particular the LC column; after several injections of the pooled sample $(\sim 5)$, chromatography conditions stabilize, improving reproducibility of the ensuing measurements (Dunn et al., 2011; Want et al., 2010). Second, the pooled sample is used to assess variability within a data set, and provides a means to correct the data for drifts in retention time or signal (Dunn et al., 2011). (For more details, see Longnecker et al. (in press) in this issue.) Ideally, the pooled sample is representative of all samples in the study that will be subject to statistical analyses. We create the pooled sample by mixing 292 aliquots of each extract $(\sim 45 \mu \mathrm{L})$ and inject it after every 6 sample injections. In our culture studies, samples are generally taken at several time points across the 294 growth cycle of a microorganism. The duration of the experiment is determined by 295 microbial growth rate, often ranging from days to weeks. On this time scale, the need for a pooled sample that encompasses the entire sample set may conflict with the desire 297 to filter, extract, and analyze samples rapidly after they are generated to minimize 298 metabolite degradation. Given the value of a fully representative pooled sample for 299 evaluating data quality, we choose to store the filters (and filtrates) generated at each sampling time point and later extract and analyze them together as a group after the 301 final time point. This approach affords two advantages: (1) a pooled QC sample is generated from aliquots of each metabolite extract and therefore reflects their 
303 composition; and (2) samples are analyzed in close succession, thereby reducing the

304 effects of instrument variability over time.

305 In addition to the pooled sample, we analyze a mix of metabolite standards at the

306 beginning and end of each sample set. In the untargeted method this mix is limited to a

307 set of 22 standards that preferentially ionize in either positive or negative ion modes

308 and span the LC gradient. In the targeted method, the full suite of standards is used

309 (Table 1). By monitoring for shifts in retention time, peak shape, and signal intensity,

310 the standard mix serves as a rapid performance check of the analytical system. Because

311 the metabolite standard mix is in highly aqueous solution (>95\% Milli-Q), it is not

312 injected during the analysis of a set of samples to avoid disrupting the matrix-

313 equilibrated column.

314 For studies involving microorganisms cultured in the laboratory, we also include

315 cell-free control samples (media blanks) treated identically to cultures with cells. In a

316 time course experiment, for example, we generally have a cell-free control at each time

317 point in addition to biological replicates. These control samples allow us to subtract

318 features from the data set corresponding to the growth media components and any

319 contaminants from the processing method. In marine field studies, however, there is no

320 clear way to define a cell-free control, and so such blanks are not used. Rather, we

321 include procedural blanks by processing and analyzing aliquots of the Milli-Q water 
322

323

324

325

326

327

328

329

330

331

332

333

334

335

336

337

used in the field. At a minimum, the procedural blanks allow us to detect contaminants that are by-products of the filtration and/or extraction methods.

Replicate measurements are an important means of assessing variability. We use the repeated injections of the pooled sample and metabolite standard mix to define instrument variability and thus do not typically include technical replicates in the analytical sequence. In the laboratory, we assess biological variability through the use of replicate growth flasks containing both media and cells. At least three biological replicates are preferable in order to perform statistical analyses. Figure 1 compares technical and biological variability in the untargeted method as measured by the percent of peaks shared across three replicate samples for: the metabolite standard mix in pure solvent ("Standard mix"), "Field samples A", and "Lab experiment B" (see Table S1 for sample details). The proportion (\%) of peaks shared was defined as the total number of peaks shared between the three replicates divided by the average number of peaks found in the three replicates. For the "Standard mix", three repeat injections of the metabolite standard mix were made. For "Field samples A", replicates were obtained by processing three water samples collected per day on 5 different days. For "Lab experiment B", points represent samples from three replicate growth flasks at multiple times along the growth curve of M. pusilla. From these measures of experimental variability, we find that technical variability is generally lower than biological variability. However, note that \#2 in "Field samples A" was as variable as the 
342 repeat injections of the standard mix. These results emphasize the importance of

343 biological replication in metabolomics experimental design, in order to appropriately

344 resolve treatment effects.

345 The accumulation of sample components in the ionization source (e.g. salt) can

346 reduce instrument sensitivity over time. To limit this potential source of instrument

347 variability we perform preventative maintenance in the ion source between sample sets.

348 This includes flushing the ESI probe with Milli-Q water and organic solvent and

349 changing the ion capillary tube. In addition to preventative cleaning, we randomize the

350 order in which samples are run relative to the order in which they were processed, to

351 avoid introducing systemic bias into the data (Dunn et al., 2011; Want et al., 2010).

352 We use a small set of isotopically labeled metabolite standards as a qualitative check

353 of recovery through the multi-step intracellular extraction protocol. Three standards,

354 d3-glutamic acid, d4-4-hydroxybenzoic acid, and d5-sodium taurocholate, are added to

355 the extraction solvent at the beginning of the filter extraction process. Consistency in the

356 measured response levels (peak areas) of these internal standards is then used as a

357 criterion for removing any samples from the set prior to data analysis. Table 2 shows

358 the percentage of the three deuterated standards recovered during filter extraction of

359 intracellular metabolites (Lab experiment E, Table S1). Another standard, deuterated

360 biotin, is added at the same level into each sample prior to LC-MS analysis as an 
361 autosampler injection standard, and can quickly alert the analyst to injection issues or

362 other instrumental problems.

363

\subsection{Sample Collection and Filtration}

We have developed procedures for the collection and filtration of marine samples

366 from both the field environment and laboratory cultures. Our sample volumes have

367 typically ranged from 50 to $4000 \mathrm{~mL}$, with laboratory cultures at the lower end and

368 samples from the open ocean at the higher end. Ultimately, the choice of sample volume

369 depends on the carbon concentrations required for acceptable metabolite signal levels

370 on our analytical platforms. From our culture experiments, we have found that a carbon

371 concentration of $0.3 \mathrm{mg} \mathrm{C}$ on filter is an ideal target, leading to high quality signal from

372 both high and low abundance metabolites. Filtering less carbon can still produce

373 acceptable metabolite signals, but detection of the lowest abundance metabolites

374 suffers. We use this value, $0.3 \mathrm{mg} \mathrm{C}$, to calculate a target cell number based on the type

375 of organism and its size. As an example, for coastal bacteria, $0.3 \mathrm{mg}$ C requires filtration

376 of approximately $10^{10}$ cells, assuming $30 \mathrm{fg} \mathrm{C}$ per cell (Fukuda et al., 1998). In our

377 culture studies, we count cells using microscopy or flow cytometry and adjust the

378 volume filtered to reach $\sim 10^{10}$ cells. To collect this number of cells in the surface ocean

379 where microbial concentrations are $\sim 10^{6}$ cells $/ \mathrm{mL}$, the ideal sample volume would be 10

380 L. In the field, however, there is often a trade-off between sample volume and the 
381 number of samples that can be collected and processed. During a recent field campaign,

382 for example, we limited our sample size to $4 \mathrm{~L}$ of seawater, less than the ideal estimated

383 volume, in order to obtain a sample set with comprehensive spatial and depth coverage.

384 Collecting more water, and therefore more cells, is always better for the detection of low

385 abundance metabolites, but this is not always practical given the significant time and

386 human resources required to filter and process each sample at sea.

387 Our filters of choice are either glass fiber or PTFE. We have tested various other

388 materials and have found that glass fiber and PTFE filters produce the least

389 downstream contamination for LC-MS analysis. The ideal filter pore size depends upon

390 the scientific question at hand. We have chosen different filtration methods for use in

391 the laboratory and the field, primarily for logistical reasons. In the laboratory, we use

392 gentle vacuum filtration with an all-glass apparatus that can be acid-cleaned and

393 combusted. Water is pulled though a PTFE filter (typically $0.2 \mu \mathrm{m}$ ) at vacuum pump

394 pressures less than $10 \mathrm{mmHg}$. We find that this filtration speed balances the need to

395 work quickly to limit metabolite degradation and the desire to avoid cell lysis.

396 Transporting an all-glass vacuum apparatus into the field, however, is neither

397 convenient nor practical. Furthermore, while it is possible to perform vacuum filtration

398 with a plastic apparatus, this would lead to unacceptable levels of plastic contamination

399 in the samples for mass spectrometry analysis. For these reasons, while at sea we have

400 chosen to use a peristaltic pump filtering apparatus equipped with Masterflex PharMed 
401 BPT peristaltic tubing, a PTFE filter holder, and PTFE tubing. Water is pumped through

402 both glass fiber and PTFE filters in series. Figure S1 illustrates the potential impact of

403 plastics on mass spectrometry data. The direct infusion mass spectrum of DOM

404 extracted (by the SPE method) from a seawater sample is shown in Figure S1A. The

405 prominent series of peaks spaced 44 mass units apart are characteristic of polymeric

406 interference from polyethylene glycol (PEG)(Keller et al., 2008). Such contamination is

407 absent from the spectrum shown in Figure S1B of a seawater sample collected and

408 extracted in the field using Teflon-based materials and Pharmed BPT peristaltic pump

409 tubing. To avoid contamination of the mass spectrometry data, we prefer to be as

410 conservative as possible in our use of plastics when collecting and processing water

411 samples, and use glass or PTFE materials whenever possible. We have, however, found

412 that collecting water in polycarbonate bottles can be acceptable.

\subsection{Metabolite Extraction Methods}

415 Metabolite extraction protocols for cells or tissues all have steps to quench

416 metabolism, release metabolites from cells, and remove remaining cellular material.

417 However, the choice of extraction method can have a significant impact on the number

418 of features observed in a metabolomics experiment as well as on their concentration,

419 largely due to the broad chemical and physical diversity of the metabolome. Given this

420 complexity and the heterogeneous nature of organic matter, no single extraction 
421 protocol will fully capture all metabolites. Our initial intracellular extraction tests were

422 performed with E. coli using protocols optimized for organisms grown in low saline

423 environments (Bolten et al., 2007; Winder et al., 2008 ). However, upon applying these

424 methods to marine microorganisms such as Ruegeria pomeroyi, few features were

425 distinguishable from the cell-free media controls. We found that the method developed

426 by Rabinowitz and Kimball (2007) using an ice cold $\left(-20^{\circ} \mathrm{C}\right)$ solution of acidic

427 acetonitrile/methanol/water to quench marine microbial cells resulted in the most

428 features distinct from the media controls (Figure 2).

429 In our initial experiments using this extraction protocol, we harvested cells of $T$.

430 pseudonana by centrifugation of $1.5 \mathrm{~mL}$ of culture for 30 minutes. Intracellular

431 metabolites were then extracted from the resulting cell pellet and analyzed by our

432 untargeted metabolomics method (Longnecker et al., 2015). To increase the biomass in

433 each extraction, we now use filtration to collect cells and, as expected, this has increased

434 the number of detected intracellular features. We have used this approach for cultures

435 of marine microbes as well as for field samples taken from the marine environment. We

436 can typically extract $\sim 18$ filters per day; vacuum centrifugation to reduce sample

437 volume at the end of the extraction protocol is generally the limiting step. We

438 conspicuously avoid bringing the samples to complete dryness during vacuum

439 centrifugation to limit metabolite degradation and evaporation. 
We investigated two approaches for preparing the intracellular filter extracts for LC-

441 MS analysis: 1) desalting the intracellular extract using solid phase extraction to enable

442 a head-to-head comparison with the extracellular extract, or 2) injecting the intracellular

443 fraction "as is" onto the targeted and untargeted LC-MS platforms. Neither option is

444 without concern. In the first case, the bias of solid phase extraction methods results in

445 selective metabolite loss. In the second, build-up of salt from the intracellular extracts

446 requires more frequent cleaning of the mass spectrometry system and replacement of

447 consumable parts. Whenever possible, we forgo additional SPE processing of the

448 intracellular extract. However, we have found that very large sample volumes, as in the

449 case of field samples, may require desalting of the intracellular extract.

450 We are also interested in the metabolites exuded by marine microorganisms into

451 their environment. Extraction of some kind is necessary to isolate these dilute

452 compounds from the highly saline matrix of seawater or culture media. Unlike urine,

453 seawater is not rich enough in metabolites to simply dilute prior to analysis (Want et al.,

454 2010). Furthermore, freeze-drying samples generates a salt-rich sample and compounds

455 cannot be detected due to lower abundance and excessive ionization suppression in the

456 LC-ESI MS analysis. Thus, methods to extract the extracellular metabolites must remove

457 salts while retaining organic matter, as well as concentrate metabolites, which otherwise

458 would be too dilute in the collected sample volume for detection by our methods.

459 Several approaches meet these criteria, including SPE with PPL, XAD and other resins, 
460 and reverse-osmosis/electrodialysis (RO/ED) (Aiken et al., 1992; Dittmar et al., 2008;

461 Kim et al., 2003; Vetter et al., 2007). An intercomparison study of three DOM extraction

462 methods, RO/ED, SPE with PPL, and SPE with XAD-8/4, showed that while RO/ED

463 captures more of the organic material than extraction by PPL ( $75-82 \%$ vs $61 \%$ ) it has

464 considerably lower sample throughput (Green et al., 2014). As such, the authors

465 recommend extraction by PPL when large numbers of samples are to be processed.

466 Solid phase extraction with PPL is in fact, currently the most popular choice for high-

467 throughput isolation of DOM (Arnold et al., 2014; Flerus et al., 2012; Lawson et al., 2014;

468 Roth et al., 2013). We have chosen PPL for extracellular metabolite extraction so that the

469 LC-MS metabolomics data sets from our field and laboratory samples are comparable,

470 and so that they can dovetail with other DOM measurements. We estimate the PPL

471 extraction efficiencies for organic carbon as described in Bhatia et al. (2010). Briefly, we

472 measure the DOC concentrations in bulk seawater prior to extraction and then measure

473 the DOC concentration in the resulting extract. From this, we can estimate the percent

474 of organic carbon we have recovered. Our metabolomics studies involving the marine

475 microbes T. pseudonana and Synechococcus elongatus in culture observed lower than

476 expected PPL extraction efficiencies of organic carbon (between 3 and $41 \%$, with

477 increased extraction efficiencies at later growth stages) (Fiore et al., in press; Longnecker

478 et al., 2015), compared to those measured by Dittmar et al. (2008) for marine and 
479 estuarine water samples. Our values are, however, comparable to dissolved organic

480 carbon recoveries reported from phytoplankton cultures (Becker et al., 2014).

481 Solid phase extraction isolates DOM molecules based on their chemical composition

482 and is known to be susceptible to extraction bias. The literature gives some insight into

483 compounds types that are not likely to be isolated well by PPL extraction. Hertkorn et

484 al. (2013) found that samples from the South Atlantic Ocean isolated with PPL showed

485 lower extraction efficiencies for dissolved organic nitrogen than for dissolved organic

486 carbon, with retention values ranging from $10.2 \%$ to $28.3 \%$, depending on depth. Other

487 reports found that highly polar compounds such as urea and amino acids were not

488 retained by PPL (Bronk, 2002). Similarly, DMSP, a polar sulfur-containing compound,

489 was not efficiently recovered by PPL extraction from the dissolved phase of cultures of

490 T. pseudonana (Longnecker et al., 2015). Another class of compounds, sugar alcohols

491 (polyols), showed low PPL extraction efficiencies from Atlantic and Pacific surface

492 DOM samples (Gonsior et al., 2014). In light of the extraction biases inherent in SPE

493 methods, we have conducted a thorough investigation of the PPL extraction recoveries

494 from a variety of chemical matrices of each metabolite in our targeted method. Full

495 description of these data is beyond the scope of this paper but will be presented in a

496 forthcoming publication (Johnson, Kido Soule \& Kujawinski, unpublished results). 
Selection of a chromatographic method for metabolomics is challenging due to the well-recognized chemical diversity of the metabolome. Unlike the proteome, metabolites can range widely in size, polarity, and charge. In fact, there is currently no 502 single chromatographic approach that is ideal for all compound classes (Lu et al., 2008). 503 Sample matrix also influences the choice of LC method; not all approaches are ideal for 504 marine organisms due to the presence of abundant salts. Other considerations include 505 sample throughput, processing time and instrument availability. Our approach for both targeted and untargeted metabolomics platforms aims to balance these issues. therefore chose existing LC approaches from the metabolomics literature shown to have enhanced retention for polar compounds and optimized separation parameters for each

510 using a metabolite standard mix. These methods included reversed phase

511 chromatography with ion-pairing (RIP), hydrophilic interaction chromatography

512 (HILIC), and reversed phase (RP) chromatography with a polar embedded C18 phase. 
518 agent on a Synergi Hydro RP column (Phenomenex, $150 \times 2$ mm, $4 \mu \mathrm{m}$ ), showed

519 excellent retention and separation of polar compounds with good peak quality.

520 However, TBA contaminates surfaces in the HPLC and MS systems and is difficult to

521 remove. In positive ion mode, the intense signal from TBA resulted in significant ion

522 suppression and was particularly problematic in the case of full scan MS operation of

523 the FT-ICR MS. Thus, the RIP chromatography method is an ideal choice, but only

524 feasible when dedicated instrumentation is available to operate solely in negative ion

525 mode.

526 We also tested an aminopropyl HILIC column (Phenomenex Luna NH2, $150 \times 2 \mathrm{~mm}$,

$5273 \mu \mathrm{m})$ in positive ion mode with an ammonium acetate mobile phase at high $\mathrm{pH}(\mathrm{pH}$

528 9.4), as reported by Bajad et al. (2006). These conditions resulted in good retention of

529 polar compounds, particularly amino acids, but lower peak quality in comparison to the

530 RIP method. As shown in Figure S2, we encountered unacceptably high

531 chromatographic background levels and difficulties with source cleanliness. This

532 particular HILIC method has been used to analyze metabolites from a variety of non-

533 marine sources, including E. coli, mammalian cells, bodily fluids, and tissues (Bajad et

534 al., 2006; Bennett et al., 2008; Onorato et al., 2008; Yuan et al., 2012). Thus, the high salt

535 content of our metabolite extracts may have contributed to challenges with this method.

536 Bajad et al. (2006), for example, observed ion suppression in their aminopropyl HILIC

537 method when sodium chloride was spiked at $\sim 100 \mathrm{mM}$ into a standards mix. 

improvements for polar retention over a traditional RP C18 column (Lu et al., 2006). We

541 optimized LC conditions for this column based on peak quality and signal-to-noise

542 levels. The resulting chromatography method produces overall very good peak shape

543 for most of the 92 compounds, with relatively narrow peak widths ranging from 10 to

54424 s at baseline (Figure S3). As expected with a RP method, non-retained inorganic salts

545 elute as a peak at or near the column dead volume, around 1.2 minutes. We use the

546 same LC conditions for our targeted and untargeted metabolomics methods, which

547 enables ready comparison between platforms. The chromatography is imperfect,

548 however, notably in the retention of very polar compounds; 45 out of the 92 metabolite

549 standards tested elute within the first 2 minutes (see Table 1). Salt from the sample

550 elutes from the column in this window as well, along with metabolites with the earliest

551 retention times (e.g. putrescine, spermidine, ornithine, arginine, s-adenosyl-L-

552 methionine, and thiamine). The potential consequences of co-elution are so-called

553 matrix effects, or the suppression (or enhancement) of the instrumental response due to

554 interference from matrix constituents (Niessen et al., 2006). Co-elution is a particular

555 issue for ESI due to its competitive nature; more abundant and/or strongly ionizing

556 compounds may suppress the formation of species with less abundance or lower

557 ionization efficiency. 
One way to improve chromatographic resolution, and thereby potentially reduce

matrix effects, is to reduce peak width with sub-2 $\mu \mathrm{m}$ particles in the stationary phase and ultrahigh performance liquid chromatography (UPLC). The UPLC approach has gained popularity in metabolomics studies as it can improve metabolite separation as

562 well as lead to more sensitive and faster analyses (Buescher et al., 2010; Dunn et al.,

563 2008; Evans et al., 2009; Want et al., 2010; Zelena et al., 2009). Narrow peak widths,

564 however, require mass spectrometers capable of collecting a sufficient number of data

565 points across a chromatographic peak. The triple quadrupole MS used for targeted

566 experiments has the requisite acquisition speed for UPLC measurements, but the FT-

567 ICR MS does not. Other MS platforms with fast enough acquisition speed for coupling

568 with UPLC include Orbitrap or time-of-flight (TOF) mass analyzers.

\subsection{Untargeted LC -MS Metabolomics Method}

571 untargeted metabolic profiling studies. These attributes facilitate both detection of co-

572 eluting species in a complex matrix (possibly having the same nominal mass) and

573 identification of unknown compounds. The most popular mass analyzers for this task

574 include TOF and Q-TOFs, Orbitraps, and FT-ICR. We use a hybrid instrument (LTQ-

575 FT), which combines a low resolution linear ion trap on the front end with a FT-ICR MS

576 on the back end. This design affords data-dependent MS/MS acquisition in the ion trap 
577 of the most abundant ions present in the high resolution full scan. The combination of

578 accurate parent mass and nominal mass fragmentation data is advantageous for

579 downstream data analysis and metabolite annotation using informatics tools.

580 For our untargeted metabolomics experiments we typically collect data in both

581 positive and negative ionization modes. This requires separate sample injections and

582 produces two data files per sample. Each data set may contain thousands of features.

583 For example, in an untargeted analysis of intracellular and extracellular metabolites

584 retained or exuded by T. pseudonana, we detected $>4,000$ features in negative ion mode

585 and $>9,000$ features in positive mode after subtraction of the controls (Longnecker et al.,

586 2015). Nonetheless, we find analyses in both ion modes valuable for profiling as much

587 of the metabolome as possible, given the ionization preference of most compounds for

588 one polarity or the other.

589 Figure 3 shows an example of LC/FT-MS results from T. pseudonana intracellular

590 metabolite extracts analyzed by our untargeted metabolomics method (Longnecker and

591 Kujawinski, unpublished). With our method, we are able to collect high-resolution and

592 high mass accuracy MS data for parent ions in parallel to MS/MS fragmentation spectra

593 at unit mass resolution. Both types of data are used in the downstream data analysis for

594 metabolite putative identification. We show an example of the MS/MS spectrum (Figure

$5953 \mathrm{C}$ ) corresponding to the feature indicated with the orange arrow in Figure 3A with $\mathrm{m} / \mathrm{z}$ 
596555.2846 and a retention time of 31.74 minutes. In this case, we used MetFrag (Wolf et

597 al., 2010) to compare the measured MS/MS fragments with fragments calculated in silico

598 for compounds with mass matches to the parent ion found in public databases (e.g.

599 KEGG, PubChem, ChemSpider). The structures of potential fragment matches are

600 shown in Figure 3D along with the feature putatively identified as a sulfoquinovosyl

601 monoacylglycerol with a C16:0 fatty acid tail (Figure 3E).

602

Other data processing steps include peak detection and peak alignment, the latter of

603 which is necessary to account for any retention time drift over the course of an

604 experiment. (For data analysis details see Longnecker et al. (in press) in this issue.) Once

605 processed, the LC/FT-MS dataset is a list of features. Each experimental data set may

606 contain thousands of features, though some do not correspond to metabolites of interest

607 but instead represent matrix or other background components and multiple ion

608 adducts. Here, experimental control samples such as method blanks are useful for

609 narrowing the list of features. More features are often observed in positive than

610 negative ion mode, likely due to greater variety of adducts generally formed by

611 electrospray ionization in positive mode (Huang et al., 1999). We examined the positive-

612 ion mode adducts formed in our untargeted method for 11 metabolite standards, shown

613 in Figure S4. All of the standards form the $[\mathrm{M}+\mathrm{H}]^{+}$adduct, and some metabolites are

614 also measured as other adducts, notably sodium. 
616 adduct formation in positive ion mode. In Figure 4, we compare the abundance of non-

617 deuterated biotin (extracted ion chromatogram peak area) detected as $[\mathrm{M}+\mathrm{H}]^{+}$and

$618[\mathrm{M}+\mathrm{Na}]^{+}$in three sample sets: two laboratory experiments (Lab experiment C and D, see

619 Table S1), and one set of water samples from the Atlantic Ocean (Field Samples B, see

620 Table S1). Each point within the figure is data from a single sample, and multiple

621 samples are shown from each sample set. With the exception of samples where biotin

622 levels are low, the peak areas for biotin observed as $[\mathrm{M}+\mathrm{H}]^{+}$are greater than the

623 corresponding measurement of biotin as $[\mathrm{M}+\mathrm{Na}]^{+}$, that is, the points fall above the one-

624 to-one line. Therefore, despite the salt in our samples, sodium adduct formation does

625 not overwhelm adducts formed with hydrogen, likely due to our LC conditions which

626 include the addition of formic acid to the mobile phase. The presence of both sodium

627 and hydrogen adducts can help in the feature annotation process, and we use freely

628 available informatics software to help define features with multiple adducts (e.g.

629 CAMERA, Kuhl et al., 2012).

\subsection{Targeted LC-MS Metabolomics Method}

In contrast to global profiling with untargeted metabolomics, the goal of targeted

632 metabolomics experiments is to quantify a well-defined set of known compounds. The

633 most widely used instrument for quantification in mass spectrometry is the triple 
634

635

636

637

638

639

640

641

642

643

644

645

646

647

648

649

650

651

652

653

quadrupole MS, which affords sensitive and specific measurements when operated in selected reaction monitoring (SRM) mode. In SRM mode, fragment ion(s) from a particular parent ion is identified, and this parent-product pair of $m / z$ values (called an SRM transition) is monitored throughout the LC run. The mass spectrometer scans through a list of SRM transitions and detects the product ion, generating an ion-specific chromatogram for each compound. Peak areas in these chromatograms are related to metabolite abundance and used for quantitative analysis across a sample set.

LC-MS can be used for either relative or absolute metabolite quantification. Absolute quantification requires the use of internal standards in order to account for variations of instrument response (from matrix effects, drifts over time) and for analyte losses during extraction and sample processing (Boyd et al., 2008). The ideal approach uses an isotopically labeled internal standard corresponding to each target metabolite.

However, the commercial availability and cost of such labeled standards is prohibitive for a method such as ours that seeks to quantify a large number of metabolites. We therefore use relative quantification in our targeted method, in which relative ion intensities are used to assess changes in metabolite concentrations across samples within an experiment (Crutchfield et al., 2010). A limited set of internal standards are used to monitor performance of the analytical method only; isotopically-labeled metabolites are added during the filter extraction of intracellular material and into each sample prior to LC analysis (injection standard). 

to obtain purified standards for each metabolite of interest. These standards are used in order to determine the fragments produced by a given compound (as this is not

657 typically known a priori) and to optimize collision energies and other instrument 658 parameters for producing a selected product ion. Many desired standards are not 659 commercially available and/or are costly, particularly in environmental metabolomics 660 where non-human metabolic pathways are of interest. Table 1 shows the list of 92 661 compounds monitored in our targeted method, compiled largely based on their 662 importance to central carbon and nitrogen pathways of marine microbes. This list 663 includes a variety of metabolite classes: nitrogenous bases, nucleosides/nucleotides, 664 phosphorylated sugars and amines, vitamins and derivatives, carbohydrate derivatives, 665 carboxylic acids, osmolytes and derivatives, amino acids, peptides and amino acid 666 derivatives, and intermediates in 3-C metabolism and lipid synthesis. Structural isomers abound in nature and exist for some of the metabolites in Table 1. 668 Such isomers are often indistinguishable by MS alone due to their same exact mass and 669 similar fragmentation patterns (Xu et al., 2015). Adding an orthogonal step, such as 670 chromatographic separation, may discriminate between isomers provided that they can 671 be resolved. For example, two isomers of adenosine monophosphate, 5'-AMP and 3'672 AMP, analyzed by our targeted method produce the same fragment ions but elute at 673 different retention times. By contrast, we cannot separate isomeric pairs such as 
674 leucine/isoleucine and threonine/homoserine by our current methods, and therefore 675 these isomers cannot be quantified individually. Table S2 lists isomers that may be 676 detected by our targeted method. We have not yet confirmed chromatographic 677 retention times for all compounds in this list with authentic standards to enable specific 678 quantification.

679 Included in Table 1 for each metabolite are instrumental limits of detection 680 (estimated for a signal-to-noise ratio of 3 ) and correlation coefficients $\left(R^{2}\right)$ of the 681 calibration curves determined by linear regression. Note that these values apply only to 682 the aqueous metabolite standard mix (>95\% Milli-Q) and may differ in the presence of a 683 complex chemical matrix such as seawater. Matrix effects may alter the instrument 684 response through ion suppression or enhancement of a given metabolite. As a result, we 685 would expect to see corresponding changes to calibration curve slopes and limits of 686 detection. To consider variability in standards, we calculate a Relative Standard 687 Deviation (RSD) for peaks found in three replicate injections. The RSD is defined as the 688 standard deviation in peak height divided by the mean peak height. Figure 5 gives the $689 \%$ RSD for the metabolite standard mix injected several times at the beginning and end 690 of a sample set, and for a pooled sample injected at multiple points throughout an 691 analytical run. The values in Figure 5 represent the range of \%RSD for the metabolites 692 detected in each type of sample. The median \%RSD for the metabolite standard mix is $69328.3 \%$ and for the pooled sample is $23.1 \%$. As with the variability assessment for the 
untargeted method shown in Figure 1, these results emphasize the need for replicate samples to enable statistical evaluation of changes in the level of a given metabolite. organic component identity in the injection solvent by drying down an aliquot of the

702 full metabolite standard mix and reconstituting in a 95:5 water:acetonitrile solution. We 703 did not notice a difference in retention time or peak shape or quality for the majority of 704 the standards, likely because of the similarly high aqueous nature of the diluted 705 standard solutions in the presence of acetonitrile or methanol. Interestingly, for three of 706 the metabolite standards (NAD, 5'-AMP, and s-adenosyl-L-homocysteine), there was a 707 pronounced effect on peak shape. With methanol in the injection solvent, the SRM 708 chromatogram contained two peaks at closely spaced retention times, while with 709 acetonitrile the peaks coalesced to a single peak with retention time intermediate to that 710 of the two peaks. For improved peak quality for these metabolites, we recommend 711 matching the organic composition of the standard solutions to that of the mobile phase 712 starting conditions (here, 5\% acetonitrile). 
714 limits the number of compounds that can be measured well in a single LC run. When 715 scanning over a large number of SRM events, sensitivity decreases when the scan time 716 for any individual SRM transition is too short. To mitigate this effect, we schedule SRM 717 transitions in the method such that they are scanned only when a given compound is 718 eluting. The actual number of SRM events in the method extends beyond the 92 719 standards in Table 1 because we may monitor a particular analyte in positive and 720 negative ion modes. In addition, we monitor two SRM transitions per parent ion. One 721 transition is used for quantification, chosen to maximize signal intensity and minimize 722 matrix interference; the second transition is used for metabolite confirmation. This 723 strategy adds specificity, which is particularly valuable in a complex organic matrix, 724 and reduces the potential for false positive identification. Figure S5 shows an example 725 of the MS/MS spectrum of non-deuterated biotin obtained by directly infusing the 726 standard into the TSQ-MS for optimization of SRM parameters. The two fragment ions 727 chosen to monitor for quantification and confirmation are highlighted with green and 728 orange circles, respectively.

729 We have used open-source software such as MAVEN (Clasquin et al., 2002;

730 Melamud et al., 2010) as well as the vendor-specific XCalibur, to process the targeted 731 metabolomics data, both of which contain tools for peak detection and quantification. In 732 either program, peak areas are calculated from extracted ion chromatograms (EIC), and 
733 this measure of instrument response is related to compound concentration to generate a 734 calibration curve for each analyte. Representative LC/TSQ-MS results (EICs and 735 standard curves) for compounds from the metabolite standard mix are shown in Figure 736 6. One important requirement of these initial data analysis steps is an assessment of 737 peak quality. MAVEN utilizes a machine-learning approach to provide a peak quality 738 score. Those with low scores warrant a manual review of the raw data. XCalibur does 739 not, however, provide such data quality estimates and so requires manual curation of 740 standard curves based on user-defined figures of merit (e.g. EIC peak quality, degree 741 and range of linear response). Quality-checked calibration curves for each analyte are 742 used to determine metabolite concentrations. Figure 7 shows an example of targeted 743 metabolomics data for several early-eluting compounds (retention time $<2$ minutes) 744 measured in intracellular extracts from a heterotrophic bacterium grown in seawater 745 (Lab experiment E, Table S1). For comparison, we show the peaks from the standard 746 mix. We use concentration values to assess relative changes (i.e. fold changes) in 747 metabolite abundance across the sample set.

748 As previously discussed, we expect to observe matrix effects at early LC retention 749 times when many metabolite species co-elute. We also anticipate similar matrix effects 750 arising from interference with the chemically complex background of dissolved organic 751 matter in seawater. Figure S6 illustrates the influence of these matrix effects on the 752 ionization efficiency of deuterated biotin. Untargeted metabolomics results from 
753 intracellular and extracellular extracts from Atlantic Ocean deep water are shown in

754 Figure S6A and S6C, respectively. DOM, which is extracted from the sample filtrate 755 along with extracellular metabolites of interest, appears as a broad, unresolved feature 756 centered at $\sim 10 \mathrm{~min}$ in Figure S6C. We find that high levels of DOM in the extracellular 757 extract can have a detrimental effect on the ionization of the d2-biotin standard, which 758 co-elutes with the organic matter background. The standard appears as a peak at 9.17 $759 \mathrm{~min}$ in the intracellular extract (Figure S6B), but in this case is entirely absent from the 760 extracellular extract (Figure S6D). Matrix effects are a potential concern when

761 calibration solutions made in solvent are applied to the quantitation of analytes in a 762 seawater or other complex matrix (Griffith et al., 2014). As noted above, we have 763 investigated the significance of matrix effects in our targeted metabolomics method but 764 full discussion of these results are beyond the scope of this paper (Johnson, Kido Soule, 765 \& Kujawinski, unpublished).

767 4. SUMMARY Marine microbial samples pose a particular challenge for metabolomics analyses due 769 to the chemical complexity of the environment and high levels of salt relative to organic 770 matter. We have developed metabolomics methods for the analysis of low molecular 771 weight metabolites from marine microbes. These methods include sampling and 
772 extraction protocols for intracellular and extracellular metabolites, liquid

773 chromatographic conditions that balance metabolite coverage and sample matrix issues,

774 and mass spectrometry platforms for profiling (untargeted metabolomics) and relative

775 quantification (targeted metabolomics) of the metabolome. Data from the untargeted

776 method can provide opportunities for the exploration of microbial metabolic pathways

777 and the discovery of new compounds. Results from the targeted method may provide

778 important quantitative limits on metabolic reactions in the ocean. Going forward, we

779 plan to incorporate alternate metabolite separation strategies such as UPLC, where

780 superior chromatographic resolution may lead to enhanced metabolome coverage and

781 better quantification. We hope that the analytical methods presented here, along with

782 continuing advances in the field of environmental metabolomics, can provide new

783 insights into microbe-DOM interactions.

785 5. ACKNOWLEDGEMENTS

We thank Cara Fiore for providing metabolomics data from laboratory cultures,

787 Crystal Breier and Gretchen Swarr for assistance in the lab with the metabolomics

788 experiments, Sonya Dyhrman and Harriet Alexander for the Rhode Island samples,

789 William A. Arnold for sample processing assistance, Ben Van Mooy and Justin

790 Ossolinski for the net trap samples, and the WHOI FT-MS Users' Facility for analyzing 
791 the metabolomics samples. We appreciate the constructive comments of three

792 anonymous reviewers. The field expedition providing the 'Atlantic Ocean' samples was

793 funded by the National Science Foundation (NSF OCE-1154320 to EBK and KL).

794 Instrumentation in the WHOI FT-MS facility was funded by the National Science

795 Foundation (OCE-0619608 and OCE-1058448) and by the Gordon and Betty T. Moore

796 Foundation through Grant GBMF1214 to EBK. The untargeted method development

797 was funded by NSF Chemical Oceanography (OCE-0928424) to EBK. Method

798 development for the targeted method was funded by the Gordon and Betty Moore

799 Foundation through Grant GBMF3304 to EBK. Support for WMJ came from a National

800 Defense Science and Engineering Fellowship.

801 


\section{REFERENCES}

Aiken, G.R., McKnight, D.M., Thorn, K.A. and Thurman, E.M., 1992. Isolation of hydrophilic organic acids from water using nonionic macroporous resins. Org Geochem 18, 567-573.

Arnold, W.A., Longnecker, K., Kroeger, K.D. and Kujawinski, E.B., 2014. Molecular signature of organic nitrogen in septic-impacted groundwater. Environmental Science: Processes \& Impacts 16, 2400-2407.

Bajad, S.U., Lu, W., Kimball, E.H., Yuan, J., Peterson, C. and Rabinowitz, J.D., 2006. Separation and quantitation of water soluble cellular metabolites by hydrophilic interaction chromatography-tandem mass spectrometry. Journal of Chromatography A 1125, 76-88.

Baran, R., Bowen, B.P., Bouskill, N.J., Brodie, E.L., Yannone, S.M. and Northen, T.R., 2010. Metabolite identification in Synechococcus sp. PCC 7002 using untargeted stable isotope assisted metabolite profiling. Anal Chem 82, 9034-9042.

Barton, R.H., Nicholson, J.K., Elliott, P. and Holmes, E., 2008. High-throughput 1H NMR-based metabolic analysis of human serum and urine for large-scale epidemiological studies: validation study. International Journal of Epidemiology 37, i31-i40.

Becker, J.W., Berube, P.M., Follett, C.L., Waterbury, J.B., Chisholm, S.W., DeLong, E.F. et al., 2014. Closely related phytoplankton species produce similar suites of dissolved organic matter. Frontiers in Microbiology 5.

Benner, R., 2002. Chemical composition and reactivity. In: D.A. Hansell and C.A. Carlson (Editors), Biogeochemistry of Marine Dissolved Organic Matter. Elsevier Science, San Diego CA, pp. 59-90.

Bennett, B.D., Yuan, J., Kimball, E.H. and Rabinowitz, J.D., 2008. Absolute quantitation of intracellular metabolite concentrations by an isotope ratio-based approach. Nat Protocols 3, 1299-1311.

Bennette, N.B., Eng, J.F. and Dismukes, G.C., 2011. An LC-MS-based chemical and analytical method for targeted metabolite quantification in the model cyanobacterium Synechococcus sp. PCC 7002. Anal Chem 83, 3808-3816.

Bhatia, M.P., Das, S.B., Longnecker, K., Charette, M.A. and Kujawinski, E.B., 2010. Molecular characterization of dissolved organic matter associated with the Greenland ice sheet Geochim Cosmochim Acta 74, 3768-3784.

Bolten, C.J., Kiefer, P., Letisse, F., Portais, J.-C. and Wittmann, C., 2007. Sampling for Metabolome Analysis of Microorganisms. Anal Chem 79, 3843-3849.

Boyd, R.K., Basic, C. and Bethem, R.A., 2008. Tools of the Trade I. The Classical Tools, Trace Quantitative Analysis by Mass Spectrometry. John Wiley \& Sons, Ltd, pp. 17-49.

Bronk, D.A., 2002. Dynamics of DON. In: D.A. Hansell and C.A. Carlson (Editors), Biogeochemistry of marine dissolved organic matter. Academic Press, pp. 153-247.

Buescher, J.M., Moco, S., Sauer, U. and Zamboni, N., 2010. Ultrahigh performance liquid chromatography-tandem mass spectrometry method for fast and robust quantification of anionic and aromatic metabolites. Anal Chem 82, 4403-4412.

Carlson, C.A., 2002. Production and removal processes. In: D.A. Hansell and C.A. Carlson (Editors), Biogeochemistry of marine dissolved organic matter. Academic Press, pp. 91151. 
Clasquin, M.F., Melamud, E. and Rabinowitz, J.D., 2002. LC-MS Data Processing with MAVEN: A Metabolomic Analysis and Visualization Engine, Current Protocols in Bioinformatics. John Wiley \& Sons, Inc.

Crutchfield, C.A., Lu, W., Melamud, E. and Rabinowitz, J.D., 2010. Mass spectrometry-based metabolomics of yeast. In: W. Jonathan, G. Christine and R.F. Gerald (Editors), Methods Enzymol. Academic Press, pp. 393-426.

Dittmar, T., Koch, B., Hertkorn, N. and Kattner, G., 2008. A simple and efficient method for the solid-phase extraction of dissolved organic matter (SPE-DOM) from seawater. Limnol Oceanogr Meth 6, 230-235.

Dunn, W.B., Broadhurst, D., Begley, P., Zelena, E., Francis-McIntyre, S., Anderson, N. et al., 2011. Procedures for large-scale metabolic profiling of serum and plasma using gas chromatography and liquid chromatography coupled to mass spectrometry. Nat Protocols 6, 1060-1083.

Dunn, W.B., Broadhurst, D., Brown, M., Baker, P.N., Redman, C.W.G., Kenny, L.C. et al., 2008. Metabolic profiling of serum using Ultra Performance Liquid Chromatography and the LTQ-Orbitrap mass spectrometry system. Journal of Chromatography B 871, 288-298.

Evans, A.M., DeHaven, C.D., Barrett, T., Mitchell, M. and Milgram, E., 2009. Integrated, nontargeted ultrahigh performance liquid chromatography/electrospray ionization tandem mass spectrometry platform for the identification and relative quantification of the small-molecule complement of biological systems. Anal Chem 81, 6656-6667.

Fiehn, O., 2008. Extending the breadth of metabolite profiling by gas chromatography coupled to mass spectrometry. TrAC Trends in Analytical Chemistry 27, 261-269.

Fiore, C.L., Longnecker, K., Kido Soule, M.C. and Kujawinski, E.B., in press. Release of ecologically relevant metabolites by the cyanobacterium, Synechococcus elongatus CCMP 1631. Environ Microbiol, DOI: 10.1111/1462-2920.12899.

Flerus, R., Lechtenfeld, O.J., Koch, B.P., McCallister, S.L., Schmitt-Kopplin, P., Benner, R. et al., 2012. A molecular perspective on the ageing of marine dissolved organic matter. Biogeosciences 9, 1935-1955.

Fukuda, R., Ogawa, H., Nagata, T. and Koike, I., 1998. Direct determination of carbon and nitrogen contents of natural bacterial assemblages in marine environments. Appl Environ Microbiol 64, 3352-3358.

Gonsior, M., Hertkorn, N., Conte, M.H., Cooper, W.J., Bastviken, D., Druffel, E. et al., 2014. Photochemical production of polyols arising from significant photo-transformation of dissolved organic matter in the oligotrophic surface ocean. Mar Chem 163, 10-18.

Green, N.W., Perdue, E.M., Aiken, G.R., Butler, K.D., Chen, H., Dittmar, T. et al., 2014. An intercomparison of three methods for the large-scale isolation of oceanic dissolved organic matter. Mar Chem 161, 14-19.

Griffith, D.R., Kido Soule, M.C., Matsufuji, H., Eglinton, T.I., Kujawinski, E.B. and Gschwend, P.M., 2014. Measuring free, conjugated, and halogenated estrogens in secondary treated wastewater effluent. Environ Sci Technol 48, 2569-2578.

Heal, K.R., Carlson, L.T., Devol, A.H., Armbrust, E.V., Moffett, J.W., Stahl, D.A. et al., 2014. Determination of four forms of vitamin B12 and other B vitamins in seawater by liquid 
chromatography/tandem mass spectrometry. Rapid Commun Mass Spectrom 28, 23982404.

Hertkorn, N., Harir, M., Koch, B.P., Michalke, B. and Schmitt-Kopplin, P., 2013. High-field NMR spectroscopy and FTICR mass spectrometry: powerful discovery tools for the molecular level characterization of marine dissolved organic matter. Biogeosciences 10, 1583-1624.

Huang, N., Siegel, M.M., Kruppa, G.H. and Laukien, F.H., 1999. Automation of a Fourier transform ion cyclotron resonance mass spectrometer for acquisition, analysis, and emailing of high-resolution exact-mass electrospray ionization mass spectral data. J Am Soc Mass Spectrom 10, 1166-1173.

Kaiser, K. and Benner, R., 2008. Major bacterial contribution to the ocean reservoir of detrital organic carbon and nitrogen. Limnology and Oceanography 53, 99-112.

Kaiser, K. and Benner, R., 2009. Biochemical composition and size distribution of organic matter at the Pacific and Atlantic time-series stations. Marine Chemistry 113, 63-77.

Keller, B.O., Sui, J., Young, A.B. and Whittal, R.M., 2008. Interferences and contaminants encountered in modern mass spectrometry. Anal Chim Acta 627, 71-81.

Kim, S., Simpson, A.J., Kujawinski, E.B., Freitas, M.A. and Hatcher, P.G., 2003. High resolution electrospray ionization mass spectrometry and 2D solution NMR for the analysis of DOM extracted by $\mathrm{C}_{18}$ solid phase disk. Org Geochem 34, 1325-1335.

Kind, T., Tolstikov, V., Fiehn, O. and Weiss, R.H., 2007. A comprehensive urinary metabolomic approach for identifying kidney cancer. Analytical Biochemistry 363, 185-195.

Kuhl, C., Tautenhahn, R., Böttcher, C., Larson, T.R. and Neumann, S., 2012. CAMERA: an integrated strategy for compound spectra extraction and annotation of liquid chromatography/mass spectrometry data sets. Anal Chem 84, 283-289.

Kujawinski, E.B., 2011. The impact of microbial metabolism on marine dissolved organic matter. Annu Rev Mar Sci 3, 567-599.

Lawson, E.C., Bhatia, M.P., Wadham, J.L. and Kujawinski, E.B., 2014. Continuous summer export of nitrogen-rich organic matter from the Greenland Ice Sheet inferred by ultrahigh resolution mass spectrometry. Environ Sci Technol 48, 14248-14257.

Lee, C., Wakeham, S.G. and I. Hedges, J., 2000. Composition and flux of particulate amino acids and chloropigments in equatorial Pacific seawater and sediments. Deep-Sea Res I 47, 1535-1568.

Longnecker, K., Futrelle, J., Coburn, E., Kido Soule, M.C. and Kujawinski, E.B., in press. Environmental metabolomics: databases and tools for data analysis. Mar Chem, DOI: 10.1016/j.marchem.2015.06.012

Longnecker, K., Kido Soule, M.C. and Kujawinski, E.B., 2015. Dissolved organic matter produced by Thalassiosira pseudonana. Mar Chem 168, 114-123.

Lu, W., Bennett, B.D. and Rabinowitz, J.D., 2008. Analytical strategies for LC-MS-based targeted metabolomics. Journal of Chromatography B 871, 236-242.

Lu, W., Clasquin, M.F., Melamud, E., Amador-Noguez, D., Caudy, A.A. and Rabinowitz, J.D., 2010. Metabolomic analysis via reversed-phase ion-pairing liquid chromatography coupled to a stand alone orbitrap mass spectrometer. Anal Chem 82, 3212-3221. 
Lu, W., Kimball, E. and Rabinowitz, J.D., 2006. A high-performance liquid chromatographytandem mass spectrometry method for quantitation of nitrogen-containing intracellular metabolites. J Am Soc Mass Spectrom 17, 37-50.

Melamud, E., Vastag, L. and Rabinowitz, J.D., 2010. Metabolomic analysis and visualization engine for LC-MS data. Anal Chem 82, 9818-9826.

Monton, M.R.N. and Soga, T., 2007. Metabolome analysis by capillary electrophoresis-mass spectrometry. Journal of Chromatography A 1168, 237-246.

Nagata, T., 2000. Production mechanisms of dissolved organic matter. In: D.L. Kirchman (Editor), Microbial Ecology of the Oceans. Wiley-Liss, Inc., New York, pp. 121-152.

Niessen, W.M.A., Manini, P. and Andreoli, R., 2006. Matrix effects in quantitative pesticide analysis using liquid chromatography-mass spectrometry. Mass Spectrom Rev 25, 881899.

Nordström, A., Want, E., Northen, T., Lehtiö, J. and Siuzdak, G., 2008. Multiple Ionization Mass Spectrometry Strategy Used To Reveal the Complexity of Metabolomics. Anal Chem 80, 421-429.

Onorato, J.M., Langish, R.A., Shipkova, P.A., Sanders, M., Wang, J., Kwagh, J. et al., 2008. A novel method for the determination of 1,5-anhydroglucitol, a glycemic marker, in human urine utilizing hydrophilic interaction liquid chromatography/MS3. Journal of Chromatography B 873, 144-150.

Patti, G.J., Yanes, O. and Siuzdak, G., 2012. Metabolomics: the apogee of the omics trilogy. Nat Rev Mol Cell Bio 13, 263-269.

Paul, C., Barofsky, A., Vidoudez, C. and Pohnert, G., 2009. Diatom exudates influence metabolism and cell growth of co-cultured diatom species. Mar Ecol Prog Ser 389, 61-70.

Quanbeck, S.M.M., Brachova, L., Campbell, A.A., Guan, X., Perera, A., He, K. et al., 2012. Metabolomics as a hypothesis-generating functional genomics tool for the annotation of Arabidopsis thaliana genes of "unknown function". Frontiers in Plant Science 3.

Rabinowitz, J.D. and Kimball, E., 2007. Acidic acetonitrile for cellular metabolome extraction from Escherichia coli. Anal Chem 79, 6167-6173.

Romano, S., Dittmar, T., Bondarev, V., Weber, R.J.M., Viant, M.R. and Schulz-Vogt, H.N., 2014. Exo-metabolome of Pseudovibrio sp. FO-BEG1 analyzed by ultra-high resolution mass spectrometry and the effect of phosphate limitation. PLoS ONE 9, e96038.

Roth, V.-N., Dittmar, T., Gaupp, R. and Gleixner, G., 2013. Latitude and pH driven trends in the molecular composition of DOM across a north south transect along the Yenisei River. Geochim Cosmochim Acta 123, 93-105.

Sañudo-Wilhelmy, S.A., Cutter, L.S., Durazo, R., Smail, E.A., Gómez-Consarnau, L., Webb, E.A. et al., 2012. Multiple B-vitamin depletion in large areas of the coastal ocean. Proc Natl Acad Sci USA 109, 14041-14045.

Southam, A.D., Payne, T.G., Cooper, H.J., Arvanitis, T.N. and Viant, M.R., 2007. Dynamic range and mass accuracy of wide-scan direct infusion nanoelectrospray Fourier Transform Ion Cyclotron Resonance mass spectrometry-based metabolomics increased by the spectral stitching method. Anal Chem 79, 4595-4602. 
Tolstikov, V.V. and Fiehn, O., 2002. Analysis of Highly Polar Compounds of Plant Origin: Combination of Hydrophilic Interaction Chromatography and Electrospray Ion Trap Mass Spectrometry. Analytical Biochemistry 301, 298-307.

Vetter, T.A., Perdue, E.M., Ingall, E., Koprivnjak, J.F. and Pfromm, P.H., 2007. Combining reverse osmosis and electrodialysis for more complete recovery of dissolved organic matter from seawater. Sep Purif Technol 56, 383-387.

Viant, M.R., 2007. Metabolomics of aquatic organisms: the new 'omics' on the block. Mar Ecol Prog Ser 332, 301-306.

Want, E.J., Nordstrom, A., Morita, H. and Siuzdak, G., 2007. From Exogenous to Endogenous: The Inevitable Imprint of Mass Spectrometry in Metabolomics. J Proteome Res 6, 459468.

Want, E.J., Wilson, I.D., Gika, H., Theodoridis, G., Plumb, R.S., Shockcor, J. et al., 2010. Global metabolic profiling procedures for urine using UPLC-MS. Nat Protocols 5, 1005-1018.

Wikoff, W.R., Anfora, A.T., Liu, J., Schultz, P.G., Lesley, S.A., Peters, E.C. et al., 2009. Metabolomics analysis reveals large effects of gut microflora on mammalian blood metabolites. Proc Natl Acad Sci USA 106, 3698-3703.

Winder, C.L., Dunn, W.B., Schuler, S., Broadhurst, D., Jarvis, R., Stephens, G.M. et al., 2008. Global metabolic profiling of Escherichia coli cultures: an evaluation of methods for quenching and extraction of intracellular metabolites. Anal Chem 80, 2939-2948.

Wolf, S., Schmidt, S., Muller-Hannemann, M. and Neumann, S., 2010. In silico fragmentation for computer assisted identification of metabolite mass spectra. BMC Bioinformatics 11, 148.

Xu, Y.-F., Lu, W. and Rabinowitz, J.D., 2015. Avoiding Misannotation of In-Source Fragmentation Products as Cellular Metabolites in Liquid Chromatography-Mass Spectrometry-Based Metabolomics. Anal Chem 87, 2273-2281.

Yanes, O., Tautenhahn, R., Patti, G.J. and Siuzdak, G., 2011. Expanding coverage of the metabolome for global metabolite profiling. Anal Chem 83, 2152-2161.

Yuan, M., Breitkopf, S.B., Yang, X. and Asara, J.M., 2012. A positive/negative ion-switching, targeted mass spectrometry-based metabolomics platform for bodily fluids, cells, and fresh and fixed tissue. Nat Protocols 7, 872-881.

Zelena, E., Dunn, W.B., Broadhurst, D., Francis-McIntyre, S., Carroll, K.M., Begley, P. et al., 2009. Development of a Robust and Repeatable UPLC-MS Method for the Long-Term Metabolomic Study of Human Serum. Anal Chem 81, 1357-1364. 


\section{Figure Captions}

1004 Figure 1. Boxplots presenting the percent of peaks shared across three replicates

1005 analyzed with the untargeted method as a measure of technical and biological

1006 variability. Table S1 provides the details on Lab experiment B and Field samples A. For

1007 the metabolite standard mix ('standard mix'), the three replicates are replicate injections

1008 of the same standard mix at different times during the LC run (technical variability).

1009 Field samples A includes three samples processed per day on five different sampling

1010 days (biological variability). Lab experiment B includes individual samples from three

1011 replicate growth flasks at multiple times along a growth curve (biological variability).

1012 Figure 2. Data from extraction protocols tested using cultures of Ruegeria pomeroyi, one

1013 method described by Rabinowitz and Kimball (2007) and a second method described by

1014 Winder et al. (2008). The data are from Lab experiment A described in Table S1. The

1015 extracts were processed immediately following the onset of the experiments at day 0 ,

1016 with subsequent extractions at days 2,3, and 5. Note that no samples were processed

1017 for day zero with the Winder et al. protocol. The y-axis is the percent increase in the

1018 features in the treatments with R. pomeroyi compared to the features observed in the

1019 cell-free controls.

1020 Figure 3. Overview of putative identification scheme. Representative LC/FT-MS

1021 chromatograms from the analysis of T. pseudonana intracellular metabolite extracts by

1022 untargeted metabolomics for (A) a sample with cells, and (B) a cell-free control. (C) is 
1023 the MS/MS spectrum collected for the peak indicated with an arrow in (A). (D) shows

1024 potential matches between the measured MS/MS fragments and in silico fragments

1025 calculated in MetFrag (Wolf et al., 2010). The compound was putatively identified as a

1026 sulfoquinovosyl monoacylglycerol with a C16:0 fatty acid (Longnecker and Kujawinski,

1027 unpublished).

1028 Figure 4. EIC peak areas for non-deuterated biotin detected as $[\mathrm{M}+\mathrm{H}]^{+}$and $[\mathrm{M}+\mathrm{Na}]^{+}$in

1029 three sample sets: two laboratory experiments (Lab experiment C (diamonds) and D

1030 (squares), see Table S1) and one set of water samples from the Atlantic Ocean (Field

1031 samples B (stars), see Table S1). The data are plotted on a log scale due to the large

1032 range of EIC peak areas and a 1:1 line is provided.

1033 Figure 5. Peak area reproducibility for the targeted method, \%RSD, calculated from

1034 replicate injections of the metabolite standard mix $(n=90)$ and of a pooled sample $(n=$ $103549)$.

1036 Figure 6. Representative LC/TSQ-MS results for selected compounds from the

1037 metabolite standard mix analyzed with the targeted metabolomics method. Extracted

1038 ion chromatograms (EIC) and calibration curves used for quantification are shown for:

1039 betaine, s-adenosyl-L-homocysteine, phenylalanine, 4-hydroxybenzoic acid, and

1040 riboflavin. Insets provide metabolite structures.

1041 Figure 7. Early eluting intracellular metabolites (retention time $<2$ minutes) from a

1042 standard mix (top row), and from an intracellular extract of a heterotrophic marine 
1043 bacterium (Samples 1 and 2, in rows 2 and 3, respectively) and analyzed with the 1044 targeted metabolomics method (Lab experiment E, Table S1). The normalization levels 1045 (NL) for each peak represent the instrument response of that metabolite in pure solvent 1046 (Row 1) or in a sample matrix (Rows 2 and 3). 
Figure 1.

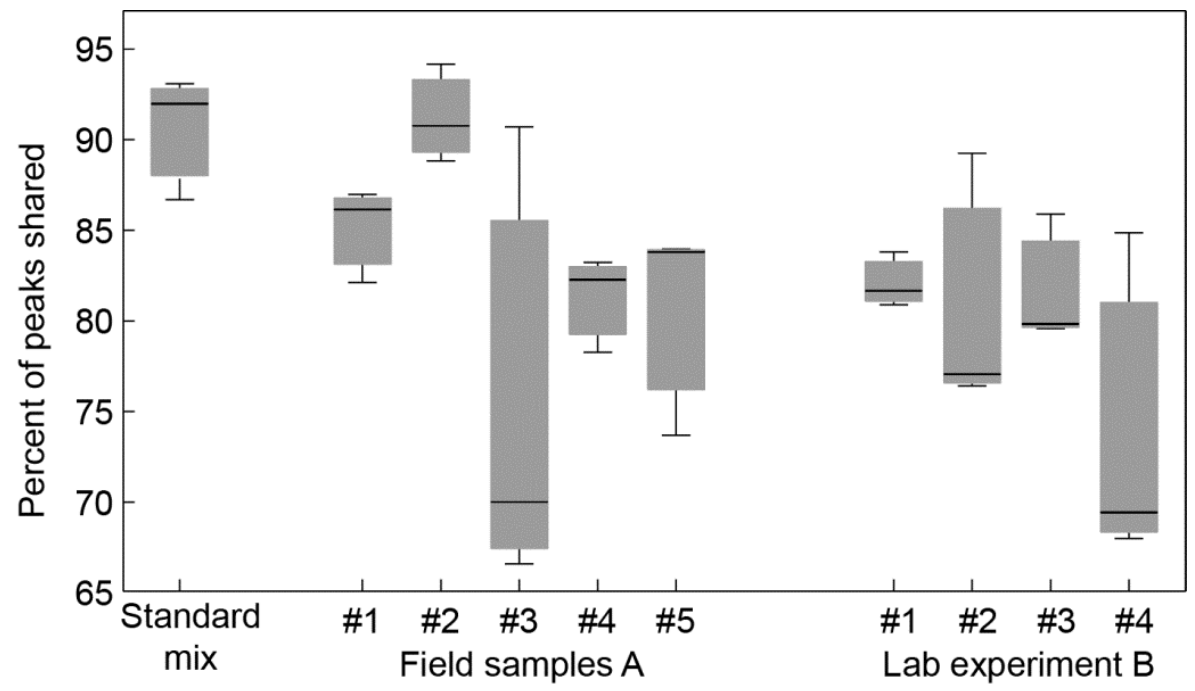

1050

1051 
1052 Figure 2.

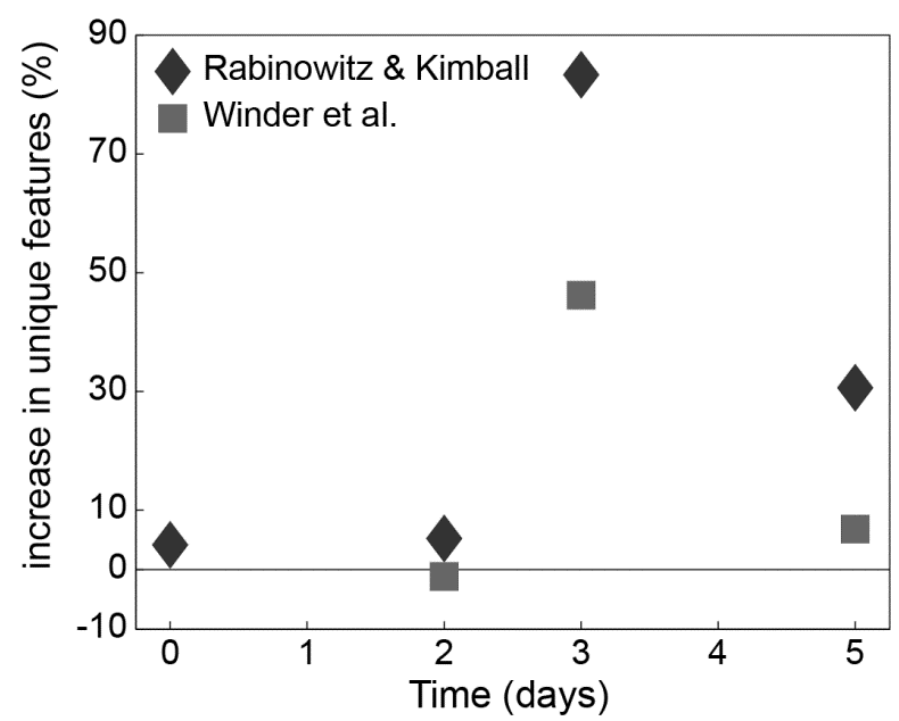

1053

1054 
Figure 3.

1056

A

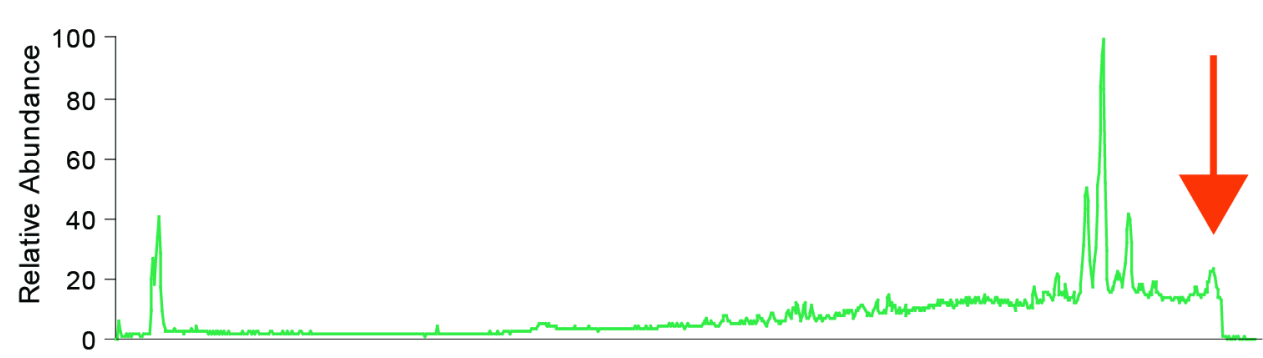

B
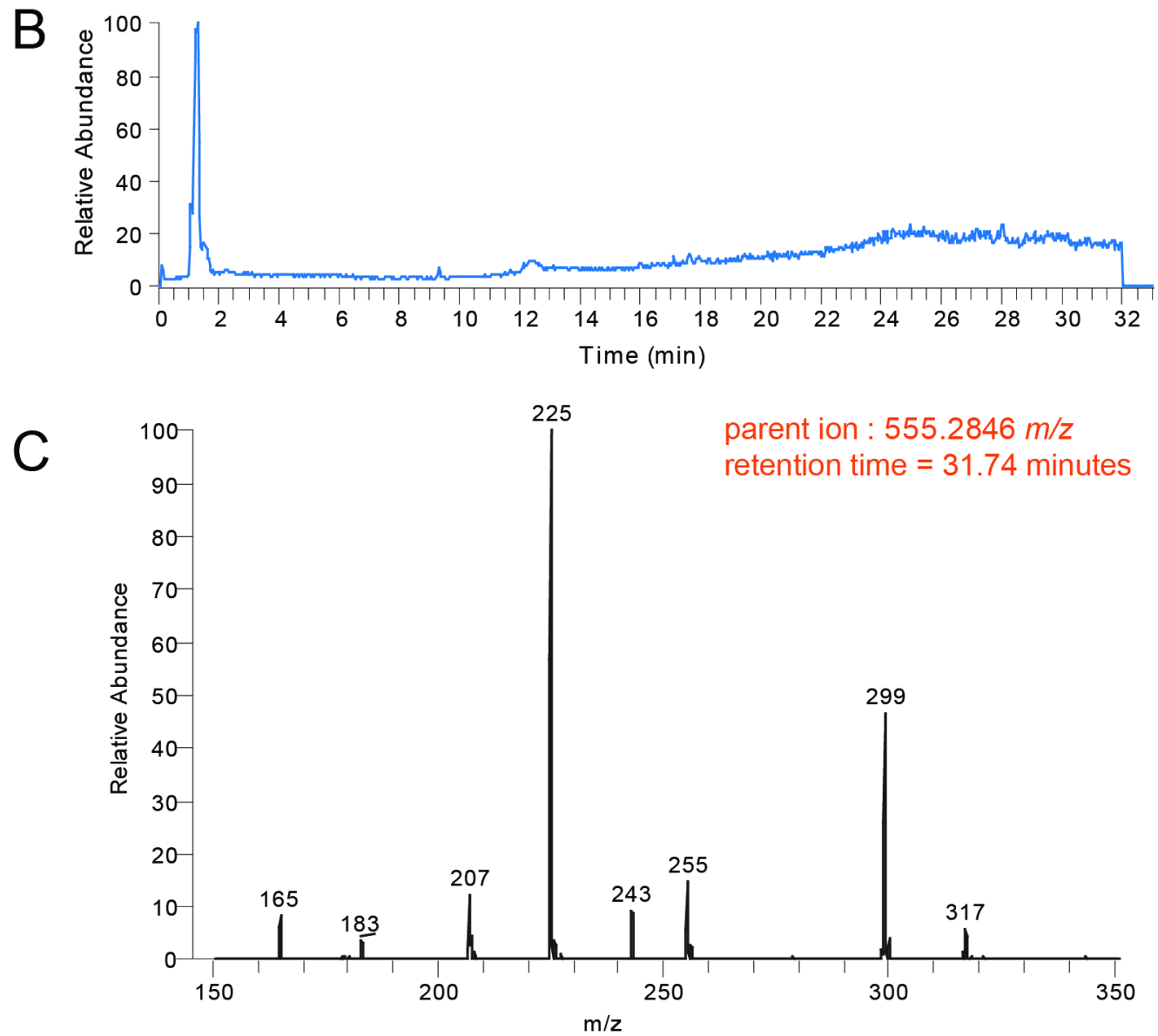

D
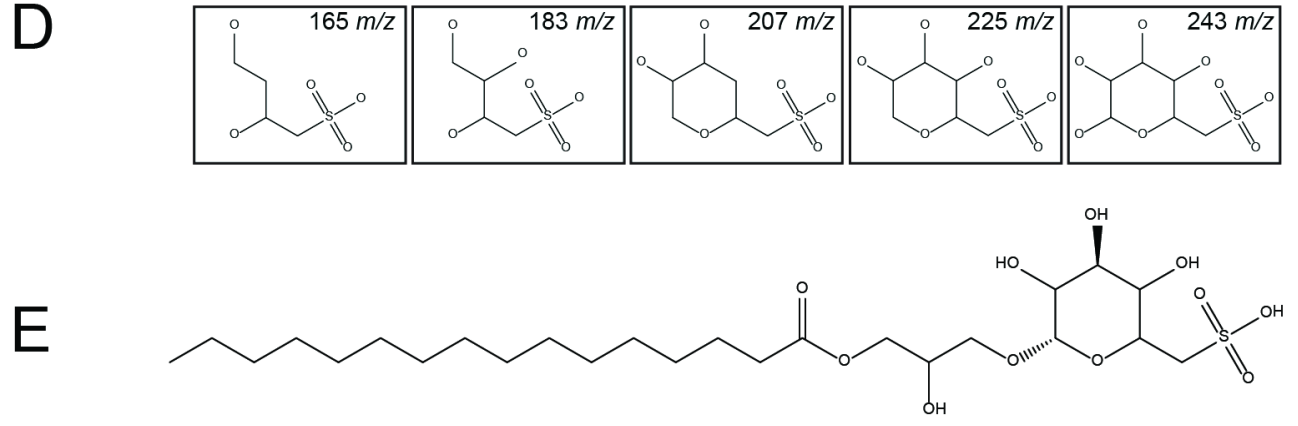
1057 Figure 4.

1058

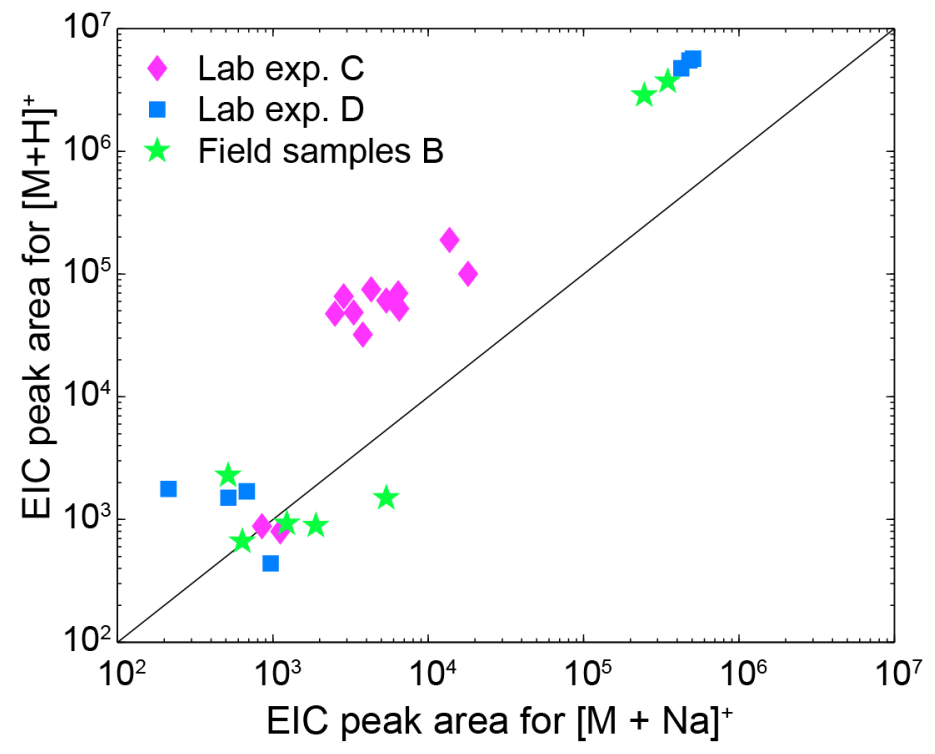

1059 
Figure 5.

1061

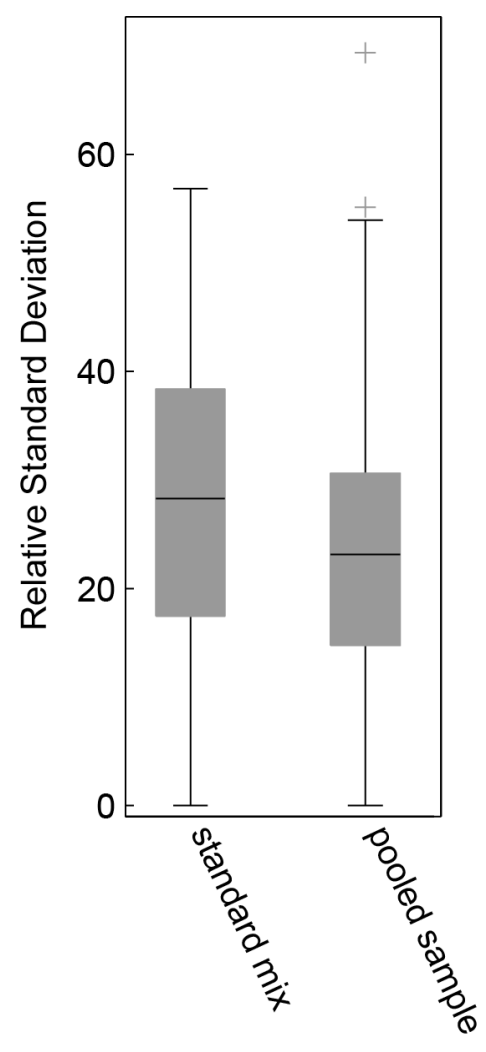

1062 
Figure 6.

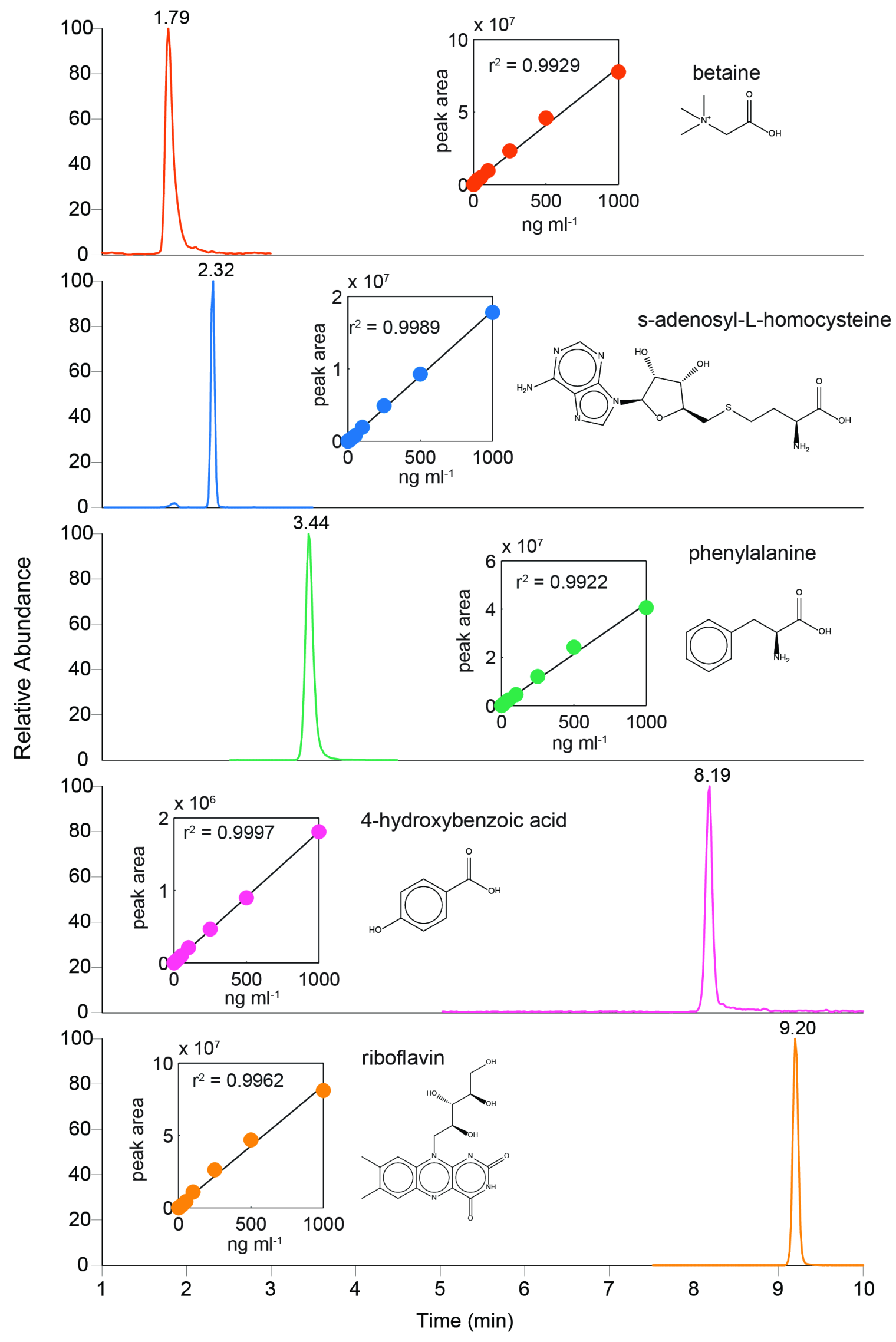




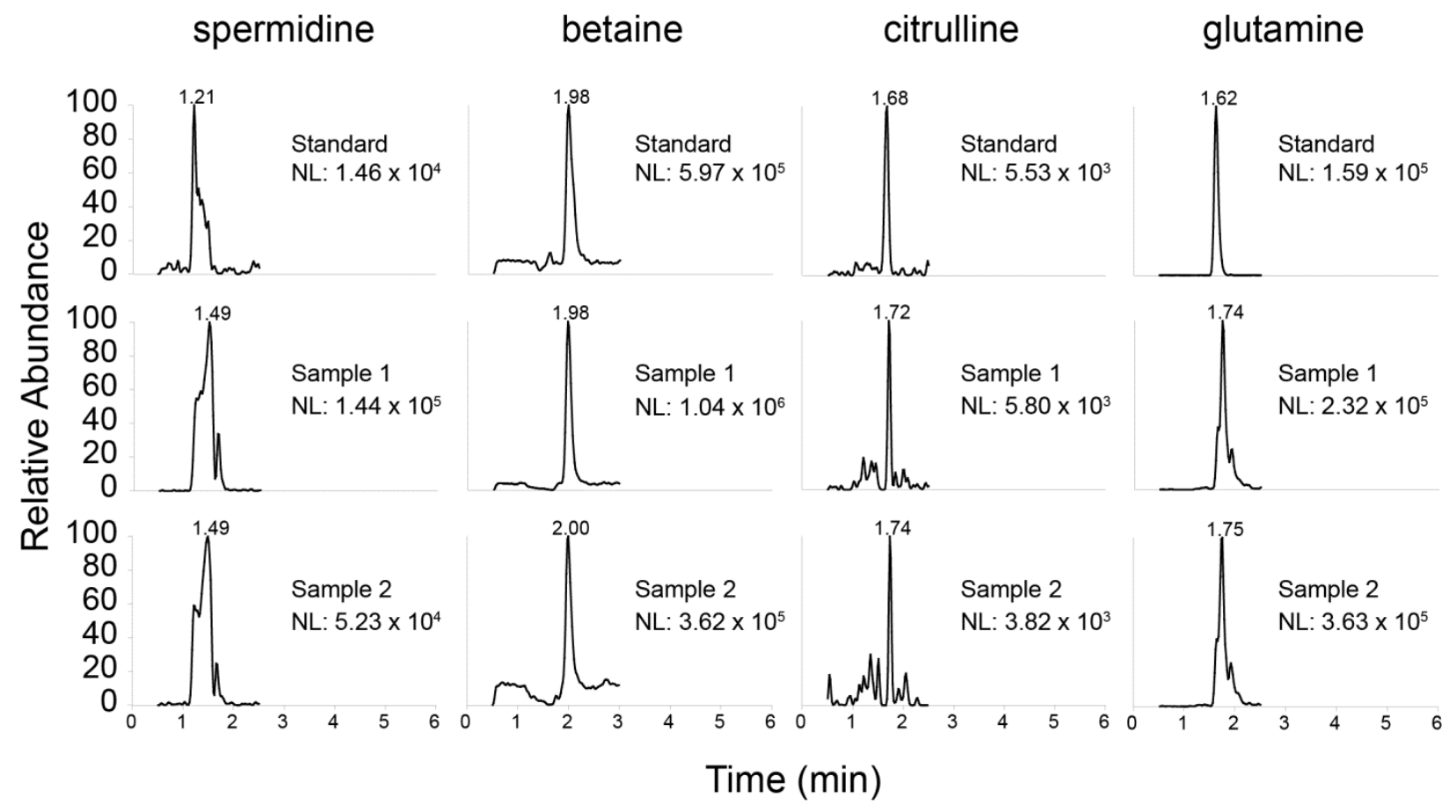

1065

1066

1067 
1068 Table 1. Compounds measured by the targeted LC-MS method in order of retention time.

\begin{tabular}{|c|c|c|c|c|c|c|c|c|}
\hline Metabolite & $\begin{array}{c}\text { RT } \\
(\mathrm{min})\end{array}$ & $\begin{array}{c}\text { Parent } \\
(\mathrm{m} / \mathrm{z})\end{array}$ & $\begin{array}{l}\text { Product }^{\mathrm{b}} \\
(\mathrm{m} / z)\end{array}$ & Polarity & $\begin{array}{l}\text { S-lens } \\
(\mathrm{V})\end{array}$ & $\begin{array}{l}C E^{c} \\
(\mathrm{~V}) \\
\end{array}$ & $\begin{array}{c}\text { Correlation } \\
\text { coefficient } \\
\left(\mathbf{R}^{2}\right) \\
\end{array}$ & $\begin{array}{c}\mathrm{LOD}^{\mathrm{d}}(\mathbf{n g} \\
\left.\mathrm{ml}^{-1}\right)\end{array}$ \\
\hline putrescine & 1.20 & 89 & $72 / 30$ & + & 30 & $5 / 24$ & 0.9912 & 100 \\
\hline spermidine & 1.20 & 146 & $72 / 112$ & + & 46 & $14 / 12$ & 0.9415 & 100 \\
\hline ornithine & 1.28 & 133 & $70 / 116$ & + & 41 & $15 / 5$ & 0.9981 & 10 \\
\hline s-adenosyl-L-methionine & 1.37 & 399 & $250 / 136$ & + & 94 & $14 / 29$ & 0.9823 & 5 \\
\hline thiamine & 1.37 & 265 & $122 / 144$ & + & 61 & $14 / 13$ & 0.9795 & 10 \\
\hline arginine & 1.38 & 175 & $70 / 130$ & + & 63 & $21 / 12$ & 0.9971 & 5 \\
\hline cytosine & 1.45 & 112 & $95 / 40$ & + & 54 & $14 / 47$ & 0.9979 & 5 \\
\hline D-glucosamine 6-phosphate & 1.49 & 258 & $79 / 97$ & - & 65 & $41 / 19$ & 0.9944 & 1 \\
\hline thiamine monophosphate & 1.49 & 345 & $122 / 126$ & + & 84 & $17 / 28$ & 0.9859 & 5 \\
\hline serine & 1.52 & 106 & $60 / 42$ & + & 36 & $10 / 21$ & 0.9868 & 5 \\
\hline $\begin{array}{l}\text { GABA ( } \gamma \text {-aminobutyric } \\
\text { acid) }\end{array}$ & 1.53 & 104 & $45 / 87$ & + & 40 & $26 / 11$ & 0.9979 & 5 \\
\hline glutamic acid & 1.54 & 146 & $128 / 102$ & - & 46 & $12 / 15$ & 0.9988 & 5 \\
\hline choline & 1.55 & 104 & $60 / 58$ & + & 70 & $15 / 31$ & 0.9851 & 1 \\
\hline $\begin{array}{l}\text { 2-aminoethylphosphonic } \\
\text { acid }\end{array}$ & 1.55 & 126 & $109 / 81$ & + & 58 & $14 / 21$ & 0.9884 & 5 \\
\hline glutamine & 1.55 & 147 & $84 / 56$ & + & 43 & $16 / 29$ & 0.9931 & 5 \\
\hline threonine & 1.55 & 120 & $74 / 56$ & + & 45 & $10 / 15$ & 0.9940 & 10 \\
\hline aspartic acid & 1.59 & 134 & $74 / 70$ & + & 43 & $13 / 14$ & 0.9825 & 10 \\
\hline citrulline & 1.59 & 176 & $70 / 159$ & + & 53 & $20 / 7$ & 0.9944 & 5 \\
\hline $\begin{array}{l}\text { dimethylsulfoniopropionate } \\
\text { (DMSP) }\end{array}$ & 1.59 & 135 & $63 / 45$ & + & 54 & $13 / 24$ & 0.9954 & 1 \\
\hline sarcosine & 1.59 & 90 & $44 / 30$ & + & 34 & $11 / 59$ & 0.9894 & 100 \\
\hline muramic acid & 1.60 & 250 & $89 / 43$ & - & 49 & $14 / 37$ & 0.9890 & 10 \\
\hline taurine & 1.62 & 124 & $80 / 107$ & - & 62 & $22 / 15$ & 0.9991 & 1 \\
\hline cysteine & 1.64 & 122 & $59 / 76$ & + & 35 & $20 / 14$ & 0.9906 & 25 \\
\hline adenine & 1.70 & 136 & $65 / 94$ & + & 85 & $37 / 20$ & 0.9819 & 1 \\
\hline ectoine & 1.70 & 143 & $68 / 97$ & + & 57 & $25 / 16$ & 0.9940 & 1 \\
\hline chitobiose & 1.72 & 425 & $126 / 84$ & + & 67 & $23 / 36$ & 0.9729 & 5 \\
\hline n-acetyl glucosamine & 1.72 & 222 & $138 / 96$ & + & 39 & $15 / 28$ & 0.9955 & 25 \\
\hline proline & 1.72 & 116 & $70 / 68$ & + & 44 & $14 / 29$ & 0.9955 & 5 \\
\hline chitotriose & 1.76 & 628 & $204 / 138$ & + & 113 & $20 / 36$ & 0.9913 & 5 \\
\hline pyridoxine & 1.76 & 170 & $134 / 152$ & + & 54 & $20 / 12$ & 0.9912 & 0.5 \\
\hline betaine & 1.81 & 118 & $58 / 42$ & + & 59 & $24 / 51$ & 0.9949 & 10 \\
\hline guanine & 1.81 & 152 & $135 / 110$ & + & 82 & $18 / 20$ & 0.9964 & 5 \\
\hline glyphosate & 1.82 & 168 & $79 / 150$ & - & 57 & $44 / 12$ & 0.9987 & 10 \\
\hline 5'-AMP & 1.89 & 348 & $136 / 119$ & + & 94 & $19 / 51$ & 0.9990 & 1 \\
\hline glutathione reduced & 1.89 & 308 & $76 / 84$ & + & 75 & $23 / 33$ & 0.9990 & 25 \\
\hline methionine & 1.90 & 150 & $56 / 61$ & + & 47 & $17 / 22$ & 0.9997 & 5 \\
\hline
\end{tabular}




\begin{tabular}{|c|c|c|c|c|c|c|c|c|}
\hline glucose 6-phosphate & 1.91 & 259 & $97 / 79$ & - & 75 & $17 / 49$ & 0.9942 & 1 \\
\hline malic acid & 1.91 & 133 & $115 / 71$ & - & 42 & $12 / 15$ & 0.9762 & 10 \\
\hline D-ribose 5-phosphate & 1.93 & 229 & $97 / 79$ & - & 58 & $15 / 43$ & 0.9898 & 1 \\
\hline 6-phosphogluconic acid & 1.95 & 275 & $79 / 97$ & - & 66 & $44 / 17$ & 0.9964 & 10 \\
\hline $\begin{array}{l}\text { dihydroxyacetone } \\
\text { phosphate }\end{array}$ & 1.95 & 169 & $79 / 97$ & - & 34 & $41 / 12$ & 0.9964 & 5 \\
\hline sn-glycerol 3-phosphate & 1.95 & 171 & $79 / 97$ & - & 53 & $41 / 18$ & 0.9986 & 10 \\
\hline $\begin{array}{l}\text { 2,3-dihydroxypropane-1- } \\
\text { sulfonate }\end{array}$ & 1.99 & 155 & $80 / 95$ & - & 51 & $33 / 18$ & 0.9976 & 1 \\
\hline D(-)3-phosphoglyceric acid & 2.01 & 185 & $79 / 97$ & - & 51 & $40 / 16$ & 0.9968 & 10 \\
\hline $\begin{array}{l}\text { 1-deoxy-D-xylulose-5- } \\
\text { phosphate }\end{array}$ & 2.04 & 213 & $79 / 97$ & - & 49 & $43 / 13$ & 0.9939 & 1 \\
\hline phosphoenolpyruvate & 2.08 & 167 & $79 / 63$ & - & 41 & $36 / 72$ & 0.9852 & 25 \\
\hline phosphomycin & 2.08 & 137 & $79 / 63$ & - & 52 & $40 / 16$ & 0.9995 & 1 \\
\hline $\begin{array}{l}\text { s-(5'-adenosyl)-L- } \\
\text { homocysteine }\end{array}$ & 2.30 & 385 & $136 / 88$ & + & 87 & $18 / 36$ & 0.9993 & 10 \\
\hline citric acid & 2.31 & 191 & $111 / 87$ & - & 47 & $13 / 17$ & 0.9653 & 10 \\
\hline glutathione oxidized & 2.31 & 613 & $355 / 231$ & + & 126 & $20 / 33$ & 0.9753 & 25 \\
\hline isoleucine & 2.31 & 132 & $69 / 56$ & + & 46 & $16 / 32$ & 0.9811 & 25 \\
\hline leucine & 2.31 & 132 & $41 / 39$ & + & 42 & $30 / 39$ & 0.9939 & 5 \\
\hline NAD & 2.31 & 664 & $136 / 428$ & + & 113 & $40 / 25$ & 0.9884 & 5 \\
\hline $\begin{array}{l}\text { s-(1,2- } \\
\text { dicarboxyethyl)glutathione }\end{array}$ & 2.31 & 424 & $259 / 214$ & + & 94 & $19 / 26$ & 0.9944 & 5 \\
\hline uracil & 2.31 & 113 & $96 / 70$ & + & 57 & $15 / 15$ & 0.9799 & 25 \\
\hline uridine 5'-monophosphate & 2.31 & 323 & $79 / 96$ & - & 100 & $43 / 22$ & 0.9930 & 1 \\
\hline NADP & 2.32 & 742 & $620 / 407$ & - & 107 & $17 / 32$ & 0.9955 & 5 \\
\hline acetyltaurine & 2.34 & 166 & $80 / 107$ & - & 57 & $35 / 18$ & 0.9994 & 0.5 \\
\hline$\alpha$-ketoglutaric acid & 2.34 & 145 & $101 / 56$ & - & 37 & $8 / 12$ & 0.9883 & 50 \\
\hline tyrosine & 2.35 & 182 & $91 / 136$ & + & 52 & $29 / 13$ & 0.9864 & 1 \\
\hline adenosine & 2.38 & 268 & $136 / 119$ & + & 74 & $17 / 43$ & 0.9993 & 0.5 \\
\hline orotic acid & 2.39 & 155 & $111 / 42$ & - & 36 & $12 / 22$ & 0.9985 & 10 \\
\hline $\begin{array}{l}(6 \mathrm{R})-5,6,7,8- \\
\text { tetrahydrobiopterin }\end{array}$ & 2.40 & 240 & $196 / 165$ & + & 80 & $13 / 21$ & 0.9988 & 5 \\
\hline inosine 5'-monophosphate & 2.42 & 349 & $137 / 119$ & + & 72 & $14 / 46$ & 0.9986 & 5 \\
\hline succinic acid & 2.52 & 117 & $73 / 99$ & - & 42 & $13 / 12$ & 0.9955 & 25 \\
\hline n-acetyl muramic acid & 2.53 & 294 & $138 / 96$ & + & 51 & $19 / 36$ & 0.9985 & 5 \\
\hline xanthine & 2.60 & 153 & $136 / 110$ & + & 60 & $14 / 16$ & 0.9969 & 5 \\
\hline fumaric acid & 2.75 & 115 & $71 / 27$ & - & 36 & $6 / 12$ & 0.9826 & 25 \\
\hline inosine & 2.83 & 267 & $135 / 108$ & - & 98 & $23 / 42$ & 0.9994 & 5 \\
\hline guanosine & 2.84 & 284 & $152 / 135$ & + & 58 & $14 / 37$ & 0.9997 & 0.5 \\
\hline phenylalanine & 3.42 & 166 & $77 / 103$ & + & 56 & $35 / 27$ & 1.0000 & 1 \\
\hline kynurenine & 3.55 & 209 & $146 / 91$ & + & 51 & $20 / 37$ & 0.9999 & 0.5 \\
\hline xanthosine & 3.58 & 285 & $153 / 136$ & + & 50 & $11 / 31$ & 0.9993 & 0.5 \\
\hline thymidine & 4.15 & 241 & $42 / 138$ & - & 76 & $19 / 15$ & 0.9987 & 10 \\
\hline
\end{tabular}




\begin{tabular}{|l|c|c|c|c|c|c|c|c|}
\hline pantothenic acid & 4.37 & 220 & $184 / 202$ & + & 58 & $11 / 10$ & 0.9998 & 5 \\
\hline 4-aminobenzoic acid & 5.84 & 138 & $77 / 65$ & + & 71 & $19 / 25$ & 1.0000 & 0.5 \\
\hline tryptamine & 6.03 & 144 & $117 / 77$ & + & 97 & $21 / 36$ & 0.9991 & 0.5 \\
\hline tryptophan & 6.13 & 205 & $146 / 118$ & + & 51 & $16 / 25$ & 1.0000 & 5 \\
\hline $\begin{array}{l}\text { 5'-deoxy- } \\
\text { 5'(methylthio)adenosine }\end{array}$ & 6.44 & 298 & $136 / 119$ & + & 77 & $16 / 46$ & 1.0000 & 0.5 \\
\hline 4-hydroxybenzoic acid & 8.07 & 137 & $93 / 65$ & - & 48 & $17 / 29$ & 0.9997 & 10 \\
\hline folic acid & 8.19 & 442 & $295 / 176$ & + & 75 & $14 / 36$ & 0.9942 & 0.5 \\
\hline caffeine & 8.57 & 195 & $138 / 110$ & + & 91 & $18 / 22$ & 1.0000 & 1 \\
\hline 2,3-dihydroxybenzoic acid & 8.92 & 153 & $109 / 108$ & - & 59 & $17 / 25$ & 0.9925 & 5 \\
\hline biotin & 9.02 & 245 & $227 / 97$ & + & 63 & $14 / 32$ & 0.9999 & 0.5 \\
\hline riboflavin & 9.15 & 377 & $243 / 172$ & + & 112 & $23 / 34$ & 0.9992 & 0.5 \\
\hline cyanocobalamin & 9.30 & 678 & $147 / 359$ & + & 137 & $34 / 20$ & 0.9996 & 0.5 \\
\hline syringic acid & 9.31 & 197 & $182 / 123$ & - & 67 & $15 / 25$ & 0.9963 & 25 \\
\hline desthiobiotin & 10.11 & 215 & $197 / 179$ & + & 63 & $12 / 17$ & 0.9995 & 0.5 \\
\hline 3-mercapto proprionate & 10.13 & 209 & $59 / 65$ & - & 41 & $20 / 46$ & 0.9959 & 5 \\
\hline indole 3-acetic acid & 12.11 & 176 & $130 / 77$ & + & 56 & $16 / 39$ & 0.9964 & 0.5 \\
\hline sodium taurocholate & 17.35 & 514 & $124 / 107$ & - & 131 & $56 / 56$ & 0.9977 & 0.5 \\
\hline
\end{tabular}

1070 a Results shown for retention time (RT), correlation coefficient $\left(\mathrm{R}^{2}\right)$, and limit of detection (LOD) apply 1071 to metabolite standard mixtures in solvent.

1072 b Two product ions $=$ quantification/confirmation.

$1073{ }^{c}$ Optimized collision energies (CE) for quantification/confirmation ions.

$1074{ }^{\mathrm{d}}$ LOD is defined as the lowest concentration at which the signal-to-noise is at least 3. 
1076 Table 2. Percentage of deuterated standards recovered during filter extraction of intracellular

1077 metabolites. The mean and one standard deviation of triplicate analyses are reported.

Deuterated Standard \% Retained

D3-glutamic acid $103 \pm 13.0$

D4-4-hydroxybenzoic acid $93 \pm 1.4$

D5-taurocholic acid $\quad 93 \pm 1.0$

1078 